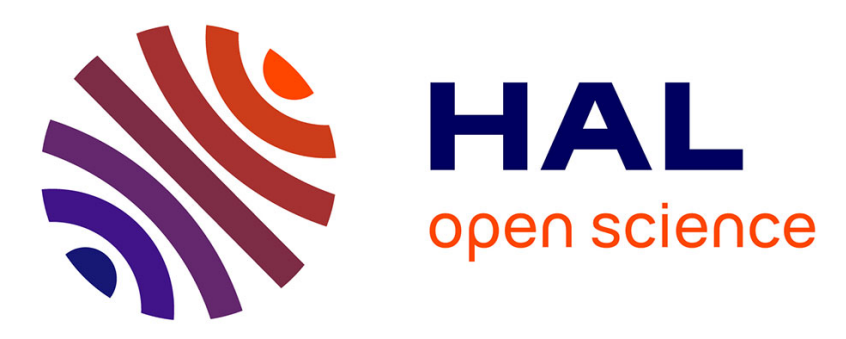

\title{
A three-dimensional conservative coupling method between an inviscid compressible flow and a moving rigid solid
}

\author{
Maria Adela Puscas, Laurent Monasse
}

\section{- To cite this version:}

Maria Adela Puscas, Laurent Monasse. A three-dimensional conservative coupling method between an inviscid compressible flow and a moving rigid solid. SIAM Journal on Scientific Computing, 2015, 37, pp.884-909. hal-00974602v2

\section{HAL Id: hal-00974602 \\ https://hal.science/hal-00974602v2}

Submitted on 1 Feb 2015

HAL is a multi-disciplinary open access archive for the deposit and dissemination of scientific research documents, whether they are published or not. The documents may come from teaching and research institutions in France or abroad, or from public or private research centers.
L'archive ouverte pluridisciplinaire HAL, est destinée au dépôt et à la diffusion de documents scientifiques de niveau recherche, publiés ou non, émanant des établissements d'enseignement et de recherche français ou étrangers, des laboratoires publics ou privés. 


\title{
A three-dimensional conservative coupling method between an inviscid compressible flow and a moving rigid solid
}

\author{
Maria Adela Puscas ${ }^{1,2,3}$ and Laurent Monasse ${ }^{1}$ \\ ${ }^{1}$ Université Paris-Est, CERMICS (ENPC), \\ 77455 Marne la Vallée cedex, France \\ email: \{puscasa, monassel\}@cermics.enpc.fr \\ 2 CEA-DAM-DIF 91297 Arpajon, France \\ email: $\{$ adela.puscas\}@cea.fr \\ ${ }^{3}$ LIMSI-CNRS, 91403 Orsay, France \\ email: $\{$ adela.puscas\}@limsi.fr
}

\begin{abstract}
We present a conservative method for the three-dimensional coupling between an inviscid compressible flow and a moving rigid solid. We consider an inviscid Euler fluid in conservative form discretized using a high-order monotonicity-preserving Finite Volume method with a directional operator splitting. An Immersed Boundary technique is employed through the modification of the Finite Volume fluxes in the vicinity of the solid. The method yields exact conservation of mass, momentum and energy of the system, and also exhibits important consistency properties, such as conservation of uniform movement of both fluid and solid as well as the absence of numerical roughness on a straight boundary. The coupling scheme evaluates the fluxes on the fluid side and the forces and torques on the solid side only once every time step, ensuring the computational efficiency of the coupling. We present numerical results assessing the robustness of the method in the case of rigid solids with large displacements.
\end{abstract}

Key Words: Fluid-structure interaction, Finite Volume, Immersed Boundary, conservative method

\section{Introduction}

A large number of engineering problems involve fluid-structure interactions. In the military or safety domains, the effects of an explosion on a building or on a submarine involve complex non-linear phenomena (shock waves, cracking, rupture, ...) $[28,30]$. The characteristic time scale of these phenomena is extremely short and the driving effect of the interaction is the overpressure. Viscous effects therefore play a lesser role in the dynamics of this type of coupled system. With an eye toward these applications, we consider in this paper an inviscid compressible flow model on the fluid side with shock waves and a rigid object on the solid side.

Fully Eulerian [13] and fully Lagrangian methods [17] have been proposed for the simulation of fluidstructure interaction. However, monolithic Eulerian or Lagrangian approaches are in general limited to the case where the fluid and the solid behave according to similar equations with different parameters (except if the regions are solved with different coupled solvers, see [3]). In this framework, the main challenges in fluid-structure interaction are the computation of the fluid forces that act on the solid and the modification of the fluid domain due to the displacement of the solid. Two main classes of methods have been developed: Arbitrary Lagrangian-Eulerian (ALE) methods and fictitious domain methods. The ALE method [8, 18] deforms the fluid domain in order to follow the movement of the structure, and this often involves possibly costly remeshing of the fluid domain when the solid goes through large displacements and especially rupture. For these reasons, we choose to use a fictitious domain method.

In fictitious domain methods, the solid is superimposed to the fixed fluid grid and additional terms are introduced in the fluid formulation to impose the fluid boundary conditions at the solid boundary. Various types of fictitious domain methods have been proposed. Non-conservative Immersed Boundary methods have been first developed for incompressible flows [7, 10, 23]. An important issue in compressible fluid-structure interaction is the conservation of mass, momentum, and energy. The accurate capture of shocks is based on conservation properties, and the preservation of physical properties is an important 
ingredient towards an effective numerical method. In addition, verifying conservation at the discrete level is a natural means to assess the numerical stability of the scheme [15]. Conservative Immersed Boundary methods $[5,11,22,27]$ and Ghost Fluid methods $[12,14]$ have been proposed for elliptic problems and compressible fluids. Conservative Immersed Boundary methods are built in such a way that the spatial discretization satisfies mass, momentum, and energy conservation. Ghost Fluid methods consist in modifying the value of ghost cells (covered by the solid) in order to compute the fluid fluxes accurately at the interface. Ghost Fluid methods often eliminate the constraint of energy conservation in order to eliminate spurious numerical oscillations at the material interface in compressible multifluid interaction problems [2].

In this work, we use the Conservative Immersed Boundary method developed in [22] in combination with a Finite Volume method (FVM) for the fluid and a Discrete Element method (DEM) for the solid. The FVM is computed on a Cartesian grid, using high-order upwind fluxes computed with a Lax-Wendroff approach [6]. The DEM $[19,21]$ is a particle method for elastodynamics, in which particles interact through forces and torques yielding the macroscopic behaviour of the assembly. Herein, the solid being rigid, is consists of a single particle. Both methods being time-explicit and computationally expensive, we develop a coupling algorithm based on an explicit time-marching procedure. The two-dimensional version of these ideas was presented in [20]. Herein, we extend the results to the three-dimensional case. This is by no means straightforward since the three-dimensional extension poses numerous challenges at the computational and algorithmic levels. The present method yields exact conservation of mass, momentum and energy of the system, and also exhibits important consistency properties, such as conservation of uniform movement of both fluid and solid as well as the absence of numerical roughness on a straight boundary. The fluid solver used in this work is formally high-order in smooth regions so as to limit numerical diffusion, but in the presence of shocks, the fluid limiters reduce the order to first order. Still the use of a high-order fluid solver is advantageous to limit numerical diffusion [6]. The solid boundary conditions in the fluid are also first order so that the coupling method is globally first order.

This paper starts in $\S 2$ with a brief description of the discretization methods for the inviscid compressible fluid and the moving rigid solid. In $\S 3$, we present the conservative coupling method based on an explicit time-marching procedure and we derive several properties of the coupling method. In $\S 4$, we describe the main geometric algorithms required for the implementation of the three-dimensional coupling scheme. In $\S 5$, we present numerical results showing in particular the energy and mass conservation achieved by the coupling scheme and the ability of the method to compute the interaction of strong discontinuities with rigid solids undergoing large displacement. Comparisons with two-dimensional numerical results are presented. Finally, conclusions are made in $\S 6$.

\section{Fluid and solid description}

The fluid is modelled by the Euler equations expressing conservation of mass, momentum, and energy for an inviscid compressible flow, which are written in Cartesian coordinates as follows:

$$
\begin{gathered}
\frac{\partial}{\partial t} U+\frac{\partial}{\partial x} F(U)+\frac{\partial}{\partial y} G(U)+\frac{\partial}{\partial z} H(U)=0, \\
U=\left(\begin{array}{c}
\rho \\
\rho u \\
\rho v \\
\rho w \\
\rho E
\end{array}\right), F(U)=\left(\begin{array}{c}
\rho u \\
\rho u^{2}+p \\
\rho u v \\
\rho u w \\
(\rho E+p) u
\end{array}\right), G(U)=\left(\begin{array}{c}
\rho v \\
\rho u v \\
\rho v^{2}+p \\
\rho v w \\
(\rho E+p) v
\end{array}\right), H(U)=\left(\begin{array}{c}
\rho w \\
\rho u w \\
\rho v w \\
\rho w^{2}+p \\
(\rho E+p) w
\end{array}\right)
\end{gathered}
$$

where $\rho$ is the mass density, $p$ the pressure, $(u, v, w)$ the Cartesian components of the velocity vector $\vec{u}$ and $E$ the total energy. The pressure in the fluid is modelled by the state equation of a perfect gas: $p=(\gamma-1) \rho e, e$ being the specific internal energy with $E=e+\frac{1}{2}\left(u^{2}+v^{2}+w^{2}\right)$ and $\gamma=1.4$ the ratio of specific heats, assumed to be constant.

The discretization of these equations is based on an explicit FVM on a Cartesian grid with directional operator splitting. For the flux calculation we use the OSMP numerical scheme which is a one-step monotonicity-preserving high-order scheme [6]. It is derived using a coupled space-time Lax-Wendroff approach, where the formal order of accuracy in the scalar case can be set to an arbitrary order. In the present work, we use order 11. The coupling method is actually independent from the numerical scheme 
used for the flux calculation. The time step, which is subjected to a CFL stability condition, is taken constant for simplicity and is denoted $\Delta t$. We introduce the discrete times $t^{n}=n \Delta t$, for all $n \geq 0$.

On the solid side, we consider a polyhedral rigid body. The solid is assumed to be star-shaped with respect to its center of mass and its faces are assumed to be star-shaped with respect to their center of mass. Working with triangular faces simplifies the geometric algorithms see $\S 4$ ). If the solid is not star-shaped, it might be preferable to decompose it into convex polyhedra when computing the geometric quantites. Thus, up to a preliminary subdivision of the polygonal faces, we consider that the solid faces are triangles. We define the thickness of the solid as the radius of the inscribed sphere. Thereafter, we assume that the solid has a thickness greater than or equal to two fluid grid cells. Various quantities are attached to the solid body, namely the position of his center of mass $\vec{X}$, the rotation matrix $\mathbf{Q}$, the velocity of the center of mass $\vec{V}$, the angular momentum matrix $\mathbf{P}$, the mass $m$, and the principal moments of inertia $I^{1}, I^{2}$ and $I^{3}$. Let $\mathbf{D}=\operatorname{diag}\left(d^{1}, d^{2}, d^{3}\right)$ with $d^{i}=\frac{1}{2}\left(I^{1}+I^{2}+I^{2}\right)-I^{i}, i \in\{1,2,3\}$. We recall the explicit solid time-integration scheme used in [20], consisting of the Verlet scheme for translation and the RATTLE scheme for rotation:

$$
\begin{aligned}
\vec{V}^{n+\frac{1}{2}} & =\vec{V}^{n}+\frac{\Delta t}{2 m} \vec{F}_{\text {fluid }}^{n}, \quad \vec{X}^{n+1}=\vec{X}^{n}+\Delta t \vec{V}^{n+\frac{1}{2}}, \\
\mathbf{P}^{n+\frac{1}{2}} & =\mathbf{P}^{n}+\frac{\Delta t}{4} \mathbf{j}\left(\overrightarrow{\mathcal{M}}_{\text {fluid }}^{n}\right) \mathbf{Q}^{n}+\frac{\Delta t}{2} \mathbf{\Upsilon}^{n} \mathbf{Q}^{n}, \\
\mathbf{Q}^{n+1} & =\mathbf{Q}^{n}+\Delta t \mathbf{P}^{n+\frac{1}{2}} \mathbf{D}^{-1}, \quad \vec{V}^{n+1}=\vec{V}^{n+\frac{1}{2}}+\frac{\Delta t}{2 m} \vec{F}_{\text {fluid }}^{n}, \\
\mathbf{P}^{n+1} & =\mathbf{P}^{n+\frac{1}{2}}+\frac{\Delta t}{4} \mathbf{j}\left(\overrightarrow{\mathcal{M}}_{\text {fluid }}^{n}\right) \mathbf{Q}^{n+1}+\frac{\Delta t}{2} \tilde{\mathbf{\Upsilon}}^{n+1} \mathbf{Q}^{n+1},
\end{aligned}
$$

where in (3), $\mathbf{\Upsilon}^{n}$ is a symmetric matrix such that

$$
\left(\mathbf{Q}^{n+1}\right)^{t} \mathbf{Q}^{n+1}=\mathbf{I},
$$

with $\mathbf{I}$ the identity matrix in $\mathbb{R}^{3}$, and in (5), $\tilde{\Upsilon}^{n+1}$ a symmetric matrix such that

$$
\left(\mathbf{Q}^{n+1}\right)^{t} \mathbf{P}^{n+1} \mathbf{D}^{-1}+\mathbf{D}^{-1}\left(\mathbf{P}^{n+1}\right)^{t} \mathbf{Q}^{n+1}=\mathbf{0},
$$

which is the constraint associated with the derivation in time of $\mathbf{Q}^{t} \mathbf{Q}=\mathbf{I}$, using the definition of $\mathbf{P}=\dot{\mathbf{Q} D}$. The matrices $\boldsymbol{\Upsilon}^{n}$ and $\tilde{\boldsymbol{\Upsilon}}^{n+1}$ are the Lagrange multipliers associated with the constraints (6) and (7), see [20]. In addition, $\vec{F}_{\text {fluid }}^{n}$ and $\overrightarrow{\mathcal{M}}_{\text {fluid }}^{n}$ denote the fluid forces and torques applied to the solid and the map $\mathbf{j}: \mathbb{R}^{3} \rightarrow \mathbb{R}^{3 \times 3}$ is such that $\mathbf{j}(\vec{x}) \vec{y}=\vec{x} \wedge \vec{y}$ for all $\vec{x}, \vec{y} \in \mathbb{R}^{3}$.

The time-integration scheme for the solid being explicit, the time step is restricted by a CFL stability condition, which is in general less stringent than the fluid CFL stability condition.

\section{Coupling method}

In the Immersed Boundary method, the solid is superimposed to the fluid grid, leading to fluid-solid mixed cells, thereafter called "cut-cells". The solid faces are collected in the set $\mathfrak{F}$, and a generic element of $\mathfrak{F}$ is denoted by $\mathcal{F}$. Owing to the movement of the solid, the solid faces, as set of points in $\mathbb{R}^{3}$, are time-dependent, and we set $\mathcal{F}^{n}=\mathcal{F}\left(t^{n}\right)$ for all $n \geq 0$. Each solid face $\mathcal{F}(t)$ is assigned a unit normal vector $\vec{\nu}_{\mathcal{F}}(t)$ (pointing from the solid to the fluid). Finally, we denote by $\Omega_{\text {solid }}(t)$ the solid domain and by $\Omega_{\text {fluid }}$ the fluid domain.

\subsection{Treatment of the cut-cells}

Recalling that we use a Cartesian grid for the fluid, we denote with integer subscripts $i, j, k$ quantities related to the center of cells and with half-integer subscripts quantities related to the center of faces of the fluid grid cells. For instance, the interface between cells $C_{i, j, k}$ and $C_{i+1, j, k}$ is denoted by $\partial C_{i+\frac{1}{2}, j, k}$. Let $C_{i, j, k}$ be a cut-cell of size $\left(\Delta x_{i, j, k}, \Delta y_{i, j, k}, \Delta z_{i, j, k}\right)$. The relevant geometric quantities describing the intersection between the moving solid and the cell $C_{i, j, k}$ are (see Fig. 1): 

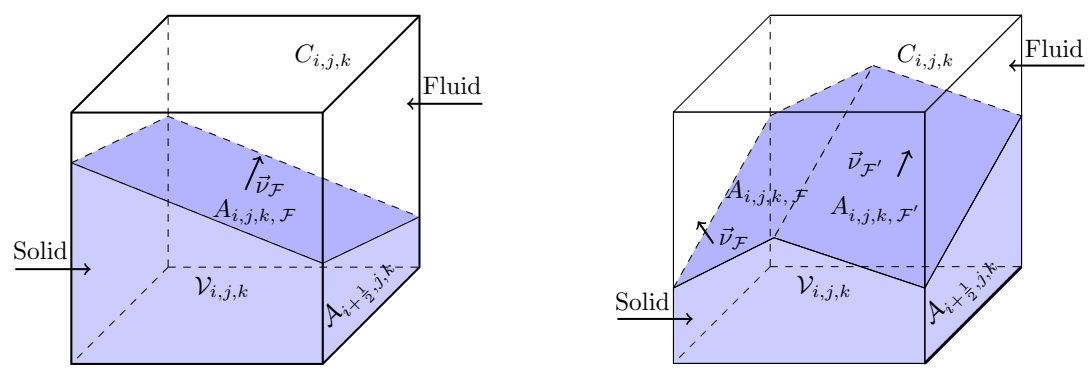

Figure 1: Two possible illustrations of a cut-cell. Left panel: the cell is intersected by one solid face. Right panel: the cell is intersected by two solid faces.

- The volume fraction $0 \leqslant \Lambda_{i, j, k}(t) \leqslant 1$ occupied by solid in $C_{i, j, k}$ at time $t$ :

$$
\Lambda_{i, j, k}(t)=\frac{\mathcal{V}_{i, j, k}(t)}{V_{i, j, k}}
$$

$V_{i, j, k}=(\Delta x \Delta y \Delta z)_{i, j, k}$ being the volume of $C_{i, j, k}$ where the solid occupies the volume $\mathcal{V}_{i, j, k}(t)$ at time $t: \mathcal{V}_{i, j, k}(t)=\int_{C_{i, j, k} \cap \Omega_{\text {solid }}(t)} d x d y d z$. When the volume fraction is evaluated at the discrete time $t^{n}$, we use the notation $\Lambda_{i, j, k}^{n}$.

- The side area fraction $0 \leqslant \lambda_{i \pm \frac{1}{2}, j, k}^{n+\frac{1}{2}}, \lambda_{i, j \pm \frac{1}{2}, k}^{n+\frac{1}{2}}, \lambda_{i, j, k \pm \frac{1}{2}}^{n+\frac{1}{2}} \leqslant 1$ of each fluid grid cell face averaged over the time interval $\left[t^{n}, t^{n+1}\right]$.

For example, on the face $\partial C_{i+\frac{1}{2}, j, k}$, we define

$$
\lambda_{i+\frac{1}{2}, j, k}^{n+\frac{1}{2}}=\frac{\mathcal{A}_{i+\frac{1}{2}, j, k}^{n+\frac{1}{2}}}{(\Delta y \Delta z)_{i, j, k}},
$$

where $\mathcal{A}_{i+\frac{1}{2}, j, k}^{n+\frac{1}{2}}=\frac{1}{\Delta t} \int_{t^{n}}^{t^{n+1}}\left(\int_{\partial C_{i+\frac{1}{2}, j, k} \cap \Omega_{\text {solid }}(t)} d y d z\right) d t$.

- The boundary area, denoted by $A_{i, j, k, \mathcal{F}}^{n+\frac{1}{2}}$, is the area of the intersection of the solid face $\mathcal{F}(t)$ with $C_{i, j, k}$ averaged over the time interval $\left[t^{n}, t^{n+1}\right]$ :

$$
A_{i, j, k, \mathcal{F}}^{n+\frac{1}{2}}=\frac{1}{\Delta t} \int_{t^{n}}^{t^{n+1}}\left(\int_{C_{i, j, k} \cap \mathcal{F}(t)} d s\right) d t .
$$

We take into account the position of the solid in the fluid domain by modifying the fluid fluxes in cut-cells. Consider such a cut-cell partially intersected by the solid, see Fig. 1 . We denote by $U_{i, j, k}^{n}$ the average value of $U$ on the fluid volume cell $C_{i, j, k}$. Integrating (1) on this cut-cell over the time interval $\left[t^{n}, t^{n+1}\right]$ and applying the divergence theorem yields (see Appendix A)

$$
\left(1-\Lambda_{i, j, k}^{n+1}\right) U_{i, j, k}^{n+1}=\left(1-\Lambda_{i, j, k}^{n}\right) U_{i, j, k}^{n}+\Delta t\left(\Phi_{i, j, k, \text { fluid }}^{n+\frac{1}{2}}+\Phi_{i, j, k, \text { solid }}^{n+\frac{1}{2}}\right),
$$

where $\Lambda_{i, j, k}^{n+1}$ is defined in (8). The fluid flux is given by

$$
\begin{aligned}
\Phi_{i, j, k, \text { fluid }}^{n+\frac{1}{2}} & =\frac{\left(1-\lambda_{i-\frac{1}{2}, j, k}^{n+\frac{1}{2}}\right)}{\Delta x_{i, j, k}} F_{i-\frac{1}{2}, j, k}^{n+\frac{1}{2}}-\frac{\left(1-\lambda_{i+\frac{1}{2}, j, k}^{n+\frac{1}{2}}\right)}{\Delta x_{i, j, k}} F_{i+\frac{1}{2}, j, k}^{n+\frac{1}{2}}+\frac{\left(1-\lambda_{i, j-\frac{1}{2}, k}^{n+\frac{1}{2}}\right)}{\Delta y_{i, j, k}} G_{i, j-\frac{1}{2}, k}^{n+\frac{1}{2}} \\
& -\frac{\left(1-\lambda_{i, j+\frac{1}{2}, k}^{n+\frac{1}{2}}\right)}{\Delta y_{i, j, k}} G_{i, j+\frac{1}{2}, k}^{n+\frac{1}{2}}+\frac{\left(1-\lambda_{i, j, k-\frac{1}{2}}^{n+\frac{1}{2}}\right)}{\Delta z_{i, j, k}} H_{i, j, k-\frac{1}{2}}^{n+\frac{1}{2}}-\frac{\left(1-\lambda_{i, j, k+\frac{1}{2}}^{n+\frac{1}{2}}\right)}{\Delta z_{i, j, k}} H_{i, j, k+\frac{1}{2}}^{n+\frac{1}{2}},
\end{aligned}
$$

where $\lambda_{i \pm \frac{1}{2}, j, k}^{n+\frac{1}{2}}, \lambda_{i, j \pm \frac{1}{2}, k}^{n+\frac{1}{2}}$, and $\lambda_{i, j, k \pm \frac{1}{2}}^{n+\frac{1}{2}}$ are defined in (9). The solid flux is given by $\Phi_{i, j, k, \text { solid }}^{n+\frac{1}{2}}=$ $\frac{1}{V_{i, j, k}} \sum_{\left\{\mathcal{F} \in \mathfrak{F} \mid \mathcal{F}^{n+1} \cap C_{i, j, k} \neq \emptyset\right\}} \phi_{i, j, k, \mathcal{F}}^{n+\frac{1}{2}}$, which results from the presence of the solid boundaries in the cell $C_{i, j, k}$. This flux takes into account the exchange of energy and momentum between the solid and the 
fluid resulting from the pressure forces.

The computation of the time-average of the side area fractions $\lambda^{n+\frac{1}{2}}$ (for simplicity, subscripts related to the fluid grid cells or faces are omitted when they play no relevant role) and of the solid flux $\phi_{\mathcal{F}}^{n+\frac{1}{2}}$ attached to $\mathcal{F}$ (involving the computation of the boundary area $A_{\mathcal{F}}^{n+\frac{1}{2}}$ ), as considered in [11], can be very complex in three dimensions. Instead of using $\Phi_{\text {fluid }}^{n+\frac{1}{2}}$ at time $n+\frac{1}{2}$, as in [20], we evaluate the side area fraction at time $t^{n+1}$, which we now denote $\Phi_{\text {fluid }}^{n+1}$, and we evaluate the solid flux, which we now denote $\Phi_{\text {solid }}^{n}$, by using the boundary area $A_{\mathcal{F}}^{n}$. This leads to the following approximation of $(10)$ :

$$
\left(1-\Lambda_{i, j, k}^{n+1}\right) U_{i, j, k}^{n+1}=\left(1-\Lambda_{i, j, k}^{n+1}\right) U_{i, j, k}^{n}+\Delta t\left(\Phi_{i, j, k, \text { fluid }}^{n+1}+\Phi_{i, j, k, \text { solid }}^{n}\right)+\Delta U_{i, j, k}^{n, n+1},
$$

where the fluid flux $\Phi_{i, j, k, \text { fluid }}^{n+1}$ is now given by

$$
\begin{aligned}
\Phi_{i, j, k, \text { fluid }}^{n+1} & =\frac{\left(1-\lambda_{i-\frac{1}{2}, j, k}^{n+1}\right)}{\Delta x_{i, j, k}} F_{i-\frac{1}{2}, j, k}^{n+\frac{1}{2}}-\frac{\left(1-\lambda_{i+\frac{1}{2}, j, k}^{n+1}\right)}{\Delta x_{i, j, k}} F_{i+\frac{1}{2}, j, k}^{n+\frac{1}{2}}+\frac{\left(1-\lambda_{i, j-\frac{1}{2}, k}^{n+1}\right)}{\Delta y_{i, j, k}} G_{i, j-\frac{1}{2}, k}^{n+\frac{1}{2}} \\
& -\frac{\left(1-\lambda_{i, j+\frac{1}{2}, k}^{n+1}\right)}{\Delta y_{i, j, k}} G_{i, j+\frac{1}{2}, k}^{n+\frac{1}{2}}+\frac{\left(1-\lambda_{i, j, k-\frac{1}{2}}^{n+1}\right)}{\Delta z_{i, j, k}} H_{i, j, k-\frac{1}{2}}^{n+\frac{1}{2}}-\frac{\left(1-\lambda_{i, j, k+\frac{1}{2}}^{n+1}\right)}{\Delta z_{i, j, k}} H_{i, j, k+\frac{1}{2}}^{n+\frac{1}{2}},
\end{aligned}
$$

the solid flux $\Phi_{i, j, k, \text { solid }}^{n}$ is now given by

$$
\Phi_{i, j, k, \text { solid }}^{n}=\frac{1}{V_{i, j, k}} \sum_{\left\{\mathcal{F} \in \mathfrak{F} \mid \mathcal{F}^{n+1} \cap C_{i, j, k} \neq \emptyset\right\}} \phi_{i, j, k, \mathcal{F}}^{n},
$$

and the so called swept amount $\Delta U_{i, j, k}^{n, n+1}=\sum_{\left\{\mathcal{F} \in \mathfrak{F} \mid \mathcal{F}^{n+1} \cap C_{i, j, k} \neq \emptyset\right\}} \Delta U_{i, j, k, \mathcal{F}}^{n, n+1}$, where $\Delta U_{\mathcal{F}}^{n, n+1}$ denotes the amount of $U$ swept by the movement of the solid face $\mathcal{F}$ during the time step from $t^{n}$ to $t^{n+1}$. The detailed procedure to compute these quantities is described in $\S 3.3$.

One possible difficulty with Immersed Boundary methods is that they can involve small cut-cells (in the sense that the solid volume fraction is greater than, say, 0.5). In order to ensure the CFL stability condition of the fluid scheme on these cells, the time step should be decreased to an unacceptably small value. To deal with this issue, we use a conservative mixing process following the ideas developed in [16]. Let $C_{p}$ be a small cell and let $C_{n}$ be a neighbouring cell with $\Lambda_{n}<\Lambda_{p}$ (see Fig. 2). We define the following exchange terms: $E_{p n}=\frac{\left(1-\Lambda_{n}\right)}{\left(2-\Lambda_{p}-\Lambda_{n}\right)}\left(U_{n}-U_{p}\right)$ and $E_{n p}=\frac{\left(1-\Lambda_{p}\right)}{\left(2-\Lambda_{p}-\Lambda_{n}\right)}\left(U_{p}-U_{n}\right)$, and we set $U_{p} \leftarrow U_{p}+E_{p n}$ and $U_{n} \leftarrow U_{n}+E_{n p}$. The mixing procedure is conservative since $\left(1-\Lambda_{p}\right) E_{p n}+\left(1-\Lambda_{n}\right) E_{n p}=0$ and ensures that the equivalent volume of a small cell is compatible with the usual CFL condition using the standard-size cells.

Another issue is the overlap of the stencil used in the FVM with the solid. Indeed, near the solid, the states needed to calculate the fluid fluxes may be located in cells completely occupied by the solid, "ghostcells" (see Fig. 3). To deal with this issue we follow the ideas developed in Ghost Fluid methods [12, 14, 29], by setting in these ghost cells a fictitious state. We define within these cells an artificial state from the states associated with the mirror cells relatively to the fluid-solid interface. Letting $C_{g}$ be a ghost cell and let $C_{m}$ be the mirror cell relatively to the fluid-solid interface, we set $\rho_{g}=\rho_{m}, p_{g}=p_{m}$, and $\vec{u}_{g}=\vec{u}_{m}-2 \vec{\nu}_{\mathcal{F}}\left(\vec{u}_{m}-\vec{V}_{\mathcal{F}}\right) \vec{\nu}_{\mathcal{F}}$, where $\mathcal{F}$ is the closest solid face in contact with the fluid and $\vec{V}_{\mathcal{F}}$ denotes the velocity of $\mathcal{F}$. This treatment possibly affects the order of the method (computation of the fluid flux) but not the conservation. One possibility to improve the order is by interpolation but it requires specific care to avoid non-physical interpolated states. Since the primary focus of this study is the conservation issue rather than the improvement of the order in the vicinity of the boundary, we have resorted to first order mirroring.

\subsection{Main steps of the coupling algorithm}

The time-integration scheme for fluid-structure interaction is based on a partitioned approach where the coupling is achieved through boundary conditions at the fluid-solid interface. In our case, for an inviscid fluid, we consider perfect slip boundary conditions at the fluid-solid interface:

$$
\vec{u}_{\text {fluid }} \cdot \vec{\nu}_{\text {fluid }}+\vec{u}_{\text {solid }} \cdot \vec{\nu}_{\text {solid }}=0, \quad \sigma_{\text {fluid }} \cdot \vec{\nu}_{\text {fluid }}+\sigma_{\text {solid }} \cdot \vec{\nu}_{\text {solid }}=0
$$




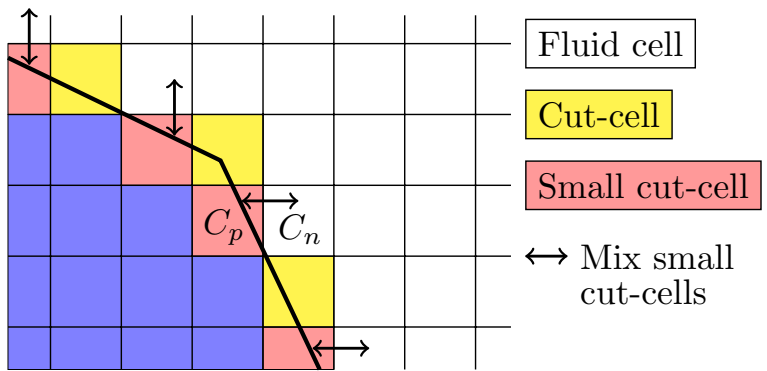

Figure 2: Mix small cut-cells

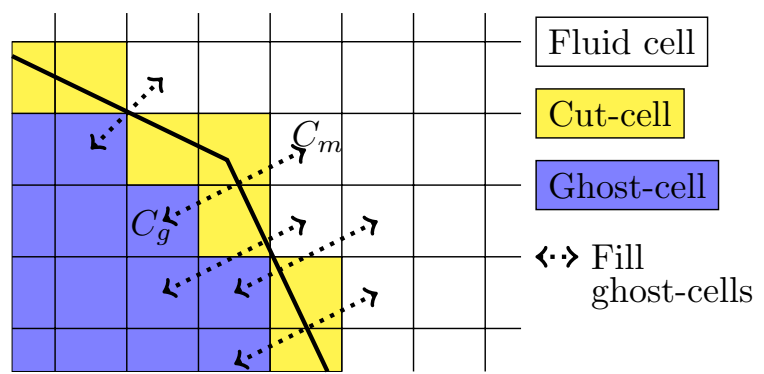

Figure 3: Fill ghost cells

where $\vec{u}_{\text {fluid }}$ and $\vec{u}_{\text {solid }}, \boldsymbol{\sigma}_{\text {fluid }}$ and $\boldsymbol{\sigma}_{\text {solid }}, \vec{\nu}_{\text {fluid }}$ and $\vec{\nu}_{\text {solid }}$ are respectively the velocities, stresses and outward pointing normals for the fluid and solid.

At the beginning of the time step from $t^{n}$ to $t^{n+1}$, we know the state of the fluid $U^{n}$, the position and rotation of the solid $\left(\vec{X}^{n}, \mathbf{Q}^{n}\right)$, as well as the velocity of its center of mass and its angular momentum $\left(\vec{V}^{n}, \mathbf{P}^{n}\right)$. The general procedure for the conservative coupling method can be described by the following five steps:

- The fluid fluxes $F^{n+\frac{1}{2}}, G^{n+\frac{1}{2}}, H^{n+\frac{1}{2}}$ are precomputed at all the cell faces of the fluid grid, without taking into account the presence of the solid. We use the one-dimensional OSMP11 scheme with directional operator splitting. For instance,

$$
U_{i, j, k}^{n+1}=L_{x}(\Delta t) L_{y}(\Delta t) L_{z}(\Delta t) U_{i, j, k}^{n}
$$

where $L_{x}, L_{y}, L_{z}$ are respectively the operators corresponding to the integration of a time step $\Delta t$ in the $x, y$ and $z$ directions. For instance, $L_{x}(\Delta t) W=W-\frac{\Delta t}{\Delta x}\left(F_{i+\frac{1}{2}, j, k}(W)-F_{i-\frac{1}{2}, j, k}(W)\right)$. Thus, secondorder time accuracy is recovered every six time steps (corresponding to all $L_{x}, L_{y}$, and $L_{z}$ permutations) if the directional operators do not commute. We denote by $\bar{p}_{x}^{n}, \bar{p}_{y}^{n}$ and $\bar{p}_{z}^{n}$ the pressures used in the application of the operators $L_{x}, L_{y}$ and $L_{z}$ respectively. These pressures are used to determine the forces exerted by the fluid on the solid during the time step.

- The fluid force $\vec{F}_{\mathcal{F}}^{n}$,fluid acting on the solid face $\mathcal{F}^{n}$ is equal to the force exerted by these pressures on the surface in contact with the fluid:

$$
\vec{F}_{\mathcal{F}, \text { fluid }}^{n}=\left(-\int_{\mathcal{F}^{n}} \bar{p}_{x}^{n} \nu_{x, \mathcal{F}}^{n},-\int_{\mathcal{F}^{n}} \bar{p}_{y}^{n} \nu_{y, \mathcal{F}}^{n},-\int_{\mathcal{F}^{n}} \bar{p}_{z}^{n} \nu_{z, \mathcal{F}}^{n}\right)^{t}
$$

The total fluid pressure force acting on the solid is the sum of the contributions on each face:

$$
\vec{F}_{\text {fluid }}^{n}=\sum_{\mathcal{F} \in \mathfrak{F}} \vec{F}_{\mathcal{F}, \text { fluid }}^{n} \text {. }
$$

The fluid torques $\overrightarrow{\mathcal{M}}_{\text {fluid }}^{n}$ are the sum of the torques of the pressure forces at the center of mass of the solid:

$$
\overrightarrow{\mathcal{M}}_{\text {fluid }}^{n}=\sum_{\mathcal{F} \in \mathfrak{F}} \vec{F}_{\mathcal{F}, \text { fluid }}^{n} \wedge\left(\vec{X}_{\mathcal{F}}^{n}-\vec{X}^{n}\right),
$$

where $\vec{X}_{\mathcal{F}}^{n}$ is the center of mass of the solid face $\mathcal{F}^{n}$ and $\vec{X}^{n}$ the center of mass of the solid.

- The solid is advanced in time. The position of the solid ( submitted to a constant external fluid force) is integrated using the Verlet scheme for translation and the RATTLE scheme for rotation (see $\S 2)$.

- The volume fractions $\Lambda^{n+1}$ and side area fractions $\lambda^{n+1}$ can then be computed using the new position of the solid boundary. The fluid fluxes are modified using $\Lambda^{n+1}, \Lambda^{n}, \lambda^{n+1}$, the pressures $\bar{p}_{x}^{n}, \bar{p}_{y}^{n}$ and $\bar{p}_{z}^{n}$ and the velocity of the boundary in order to enforce the conservation of fluid mass and of the total momentum and energy of the system. At this stage, we can also calculate the swept amount $\Delta U_{\mathcal{F}}^{n, n+1}$.

- The final value of the state $U_{i, j, k}^{n+1}$ in the cell is calculated using (11). Owing to the perfect slip conditions at the solid boundary, the flux $\phi_{\mathcal{F}}^{n}$ is given by

$$
\phi_{\mathcal{F}}^{n}=\left(0, \Pi_{x, \mathcal{F}}^{n}, \Pi_{y, \mathcal{F}}^{n}, \Pi_{z, \mathcal{F}}^{n}, \vec{V}_{\mathcal{F}}^{n+\frac{1}{2}} \cdot \vec{\Pi}_{\mathcal{F}}^{n}\right)^{t},
$$


where $\vec{\Pi}_{\mathcal{F}}^{n}=\left(\int_{\mathcal{F}^{n}} \bar{p}_{x}^{n} \nu_{x, \mathcal{F}}^{n}, \int_{\mathcal{F}^{n}} \bar{p}_{y}^{n} \nu_{y, \mathcal{F}}^{n}, \int_{\mathcal{F}^{n}} \bar{p}_{z}^{n} \nu_{z, \mathcal{F}}^{n}\right)^{t}=-\vec{F}_{\mathcal{F}, \text { fluid }}^{n}$, and $\vec{V}_{\mathcal{F}}^{n+\frac{1}{2}}$ is the velocity of the center of mass of the solid face $\mathcal{F}^{n}$ :

$$
\vec{V}_{\mathcal{F}}^{n+\frac{1}{2}}=V^{n+\frac{1}{2}}+\vec{\Omega}^{n+\frac{1}{2}} \wedge\left(\vec{X}_{\mathcal{F}}^{n}-\vec{X}^{n}\right)
$$

where $V^{n+\frac{1}{2}}$ and $\vec{\Omega}^{n+\frac{1}{2}}$ are, respectively, the average velocity and rotation velocity of the solid in the time interval $\left[t^{n}, t^{n+1}\right]$. We define the angular velocity $\vec{\Omega}^{n+\frac{1}{2}}$ at time $\left(n+\frac{1}{2}\right) \Delta t$ using the relation $\mathbf{j}\left(\vec{\Omega}^{n+\frac{1}{2}}\right)=\frac{1}{2} \mathbf{P}^{n+\frac{1}{2}} \mathbf{D}^{-1}\left(\mathbf{Q}^{n}+\mathbf{Q}^{n+1}\right)^{t}$.

We finish by mixing the small cut-cells, and we fill the ghost-cells in order to prepare the next time step (see $\S 3.1$ ). The general structure of the coupling scheme is presented in Fig. 4.

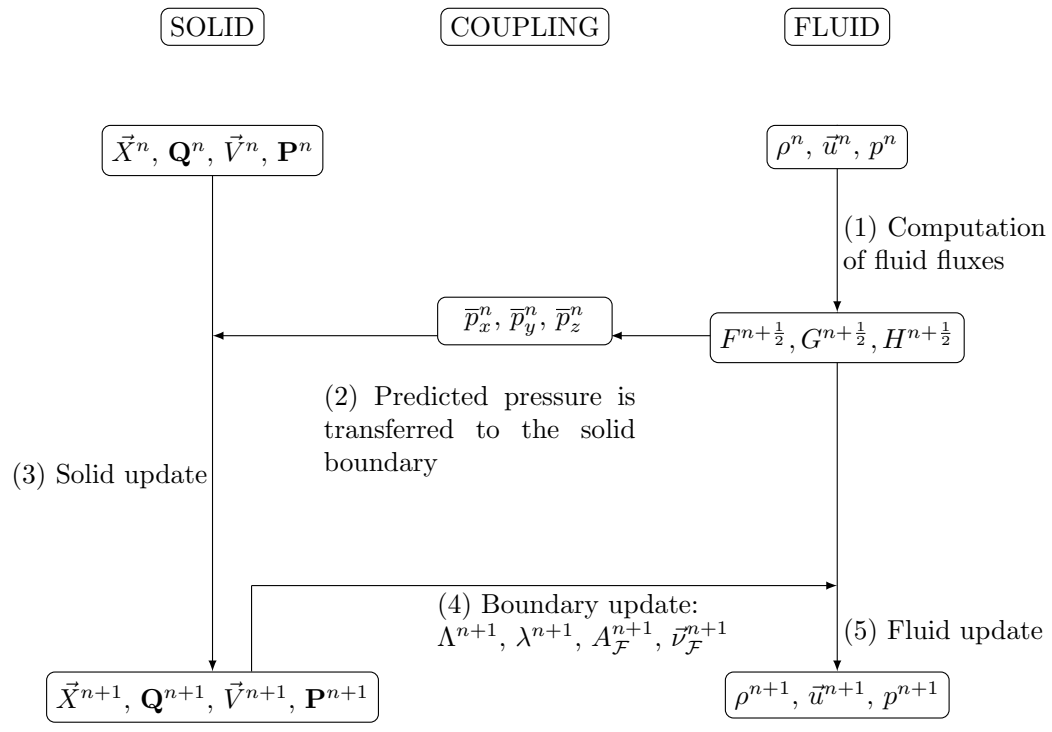

Figure 4: Structure of the coupling scheme

\subsection{Swept amount}

We now detail the computation of the amount swept by the movement of the solid interface during the time step from $t^{n}$ to $t^{n+1}$ and its distribution over the cut-cells. We first subdivide each solid face $\mathcal{F}^{n}$ and $\mathcal{F}^{n+1}$ into a set of triangles (called sub-faces) entirely contained in one cell. We then compute the amount swept by the movement of each sub-face and we attribute this amount to the cell containing the sub-face at time $t^{n+1}$.

\subsubsection{Map between $\mathcal{F}^{n}$ and $\mathcal{F}^{n+1}$ and sub-mesh}

To facilitate the computation of the swept amount $\Delta U_{\mathcal{F}}^{n, n+1}$, we subdivide each solid face $\mathcal{F}$ into a set of triangles (called sub-faces) that are contained in one fluid grid cell (not necessary the same) at times $t^{n}$ and $t^{n+1}$. We define a piecewise affine map $\Psi_{n, n+1}$ from $\mathcal{F}^{n}$ to $\mathcal{F}^{n+1}$. If a triangular sub-face has vertices $\bar{a}_{1}^{n}, \bar{a}_{2}^{n}$ and $\bar{a}_{3}^{n}$, we can express a point $x$ of the sub-face at time $t^{n}$ as the weighted combination: $x=\alpha_{1}(x) \bar{a}_{1}^{n}+\alpha_{2}(x) \bar{a}_{2}^{n}+\alpha_{3}(x) \bar{a}_{3}^{n} ; \alpha_{1}(x), \alpha_{2}(x), \alpha_{3}(x) \geq 0, \alpha_{1}(x)+\alpha_{2}(x)+\alpha_{3}(x)=1$. The local map $\Psi_{n, n+1}$ is then defined as

$$
\Psi_{n, n+1}(x)=\alpha_{1}(x) \bar{a}_{1}^{n+1}+\alpha_{2}(x) \bar{a}_{2}^{n+1}+\alpha_{3}(x) \bar{a}_{3}^{n+1} .
$$

Let us consider the case of Fig. 5: in panel 5a, we have drawn the intersection of the solid face $\mathcal{F}$ with a fluid grid cell $C_{i, j, k}$ at time $t^{n}$ and in panel $5 \mathrm{~b}$, the intersection at time $t^{n+1}$. Using the map (18), we now can draw the intersection on the same plane, see Fig. 6, where we have also drawn the whole face $\mathcal{F}$. If we now consider the intersection of $\mathcal{F}$ with all the fluid grid cells, we can obtain the result shown in Fig. 7: the intersections at time $t^{n}$ mapped by $\Psi_{n, n+1}$ are drawn in continuous lines, and the intersections at time $t^{n+1}$ in dashed lines. We denote these polygonal meshes respectively with $S_{\mathcal{F}}^{n}$ and $S_{\mathcal{F}}^{n+1}$. The idea 
is now to intersect $\Psi_{n, n+1}\left(S_{\mathcal{F}}^{n}\right)$ with $S_{\mathcal{F}}^{n+1}$. We triangulate the polygonal mesh obtained by the previous intersections at time $t^{n}$ and $t^{n+1}$ in order to build a sub-mesh of both $S_{\mathcal{F}}^{n}$ and $S_{\mathcal{F}}^{n+1}$ (see Fig.8). Thus, the solid face $\mathcal{F}$ is decomposed into a set of triangles, called sub-faces, denoted by $f$, so that $\mathcal{F}=\cup f$. As for $\mathcal{F}$, we set $f^{n}=f\left(t^{n}\right)$ for all $n \geq 0$.

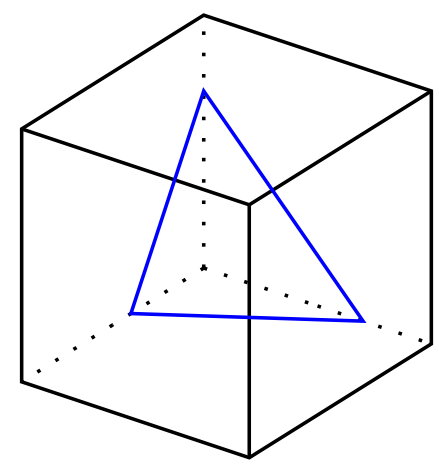

(a) $C_{i, j, k} \cap \mathcal{F}^{n}$

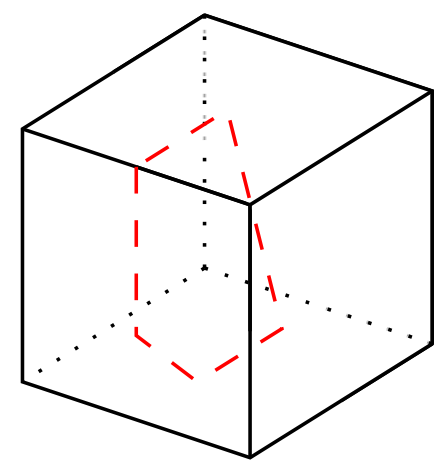

(b) $C_{i, j, k} \cap \mathcal{F}^{n+1}$

Figure 5: Intersections between a fluid cell and a solid face at time $t^{n}$ (a) and $t^{n+1}$ (b).

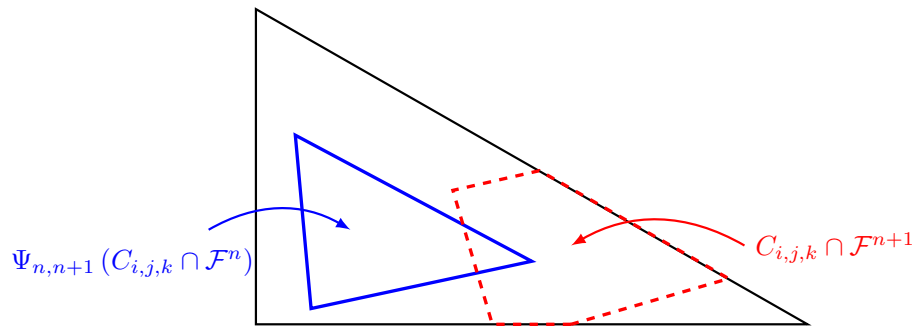

Figure 6: Intersections between a solid face and one fluid grid cell at time $t^{n}$ (continuous line) and $t^{n+1}$ (dashed line).

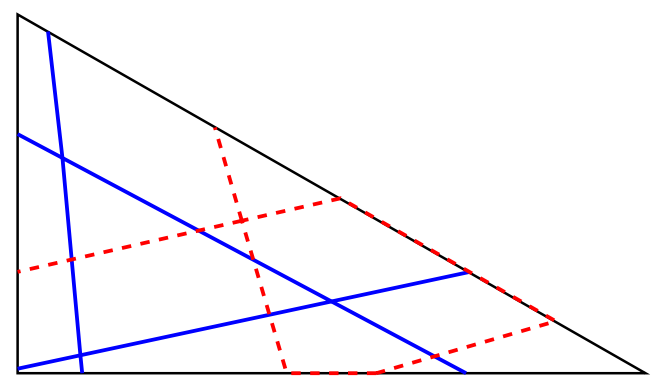

Figure 7: Position of the two intersections: the corresponding meshes at time $t^{n}$ (continuous line) and $t^{n+1}$ (dashed line).

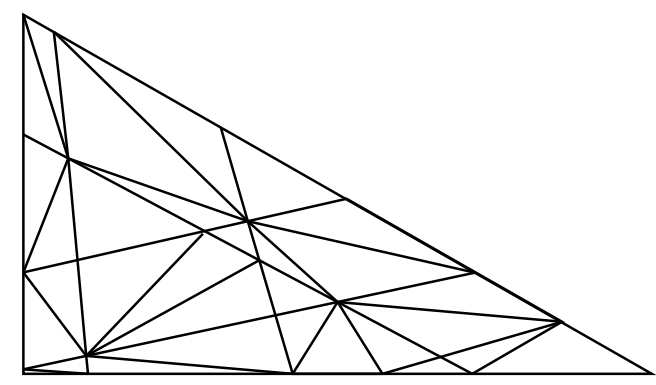

Figure 8: Triangular sub-mesh

\subsubsection{Computation of the integral over the prism and distribution over the cut-cells}

The amount swept by the sub-face $f$ during the time step from $t^{n}$ to $t^{n+1}$ assigned to the cell containing $f$ at time $t^{n+1}$, denoted $\Delta U_{i, j, k, f}^{n, n+1}$, is the integral of $U^{n}$ on the prism (possibly twisted, see $\S 4.2$ ) $K_{f}$ whose bases are $f^{n}$ and $f^{n+1}$ (see Fig. 9):

$$
\Delta U_{i, j, k, f}^{n, n+1}=\frac{1}{V_{i, j, k}} \int_{K_{f}} U\left(t^{n}, x, y, z\right) d x d y d z .
$$

Since $U^{n}$ is piecewise constant, the integral over the prism $K_{f}$ is equal to 


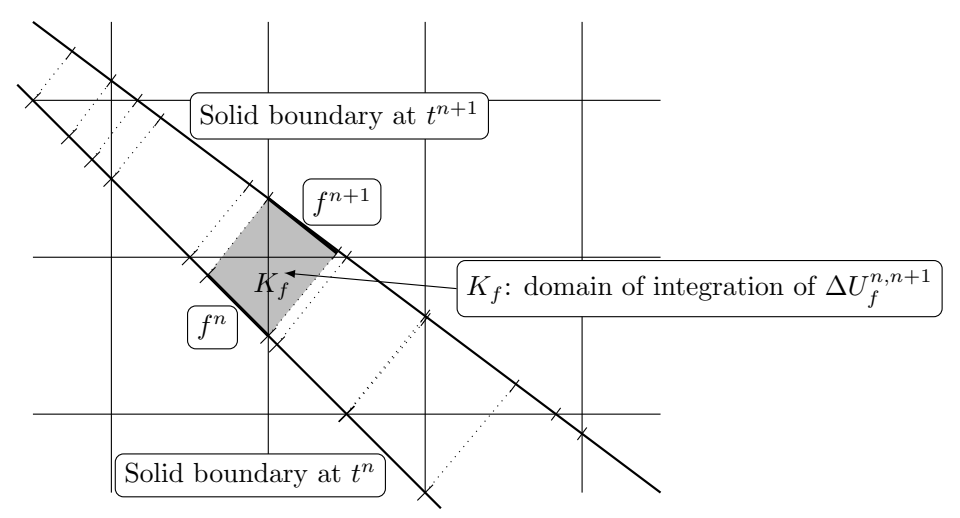

Figure 9: Two-dimensional illustration of the computation of $\Delta U_{f}^{n, n+1}$

$$
\int_{K_{f}} U\left(t^{n}, x, y, z\right) d x d y d z=\sum_{\left\{C_{p, q, r} \mid K_{f} \cap C_{p, q, r} \neq \emptyset\right\}} \mathcal{V}_{p, q, r} U_{p, q, r}^{n}
$$

where $\mathcal{V}_{p, q, r}$ is the signed volume (in the sense that it can be positive if the prism is positively oriented or negative if it is not) of the intersection between the prism $K_{f}$ and the fluid grid cell $C_{p, q, r}$ (see $\S 4.2$ for computation details). Thus, $\Delta U_{i, j, k, f}^{n, n+1}$ is given by

$$
\Delta U_{i, j, k, f}^{n, n+1}=\frac{1}{V_{i, j, k}} \sum_{\left\{C_{p, q, r} \mid K_{f} \cap C_{p, q, r} \neq \emptyset\right\}} \mathcal{V}_{p, q, r} U_{p, q, r}^{n}
$$

and, finally the swept amount assigned to the cell $C_{i, j, k}$ is the sum of the amount swept by each sub-face $f^{n+1}$ contained in the cell,

$$
\Delta U_{i, j, k}^{n, n+1}=\sum_{\left\{\mathcal{F} \in \mathfrak{F} \mid \mathcal{F}^{n+1} \cap C_{i, j, k} \neq \emptyset\right\}\left\{f \subset \mathcal{F}^{n+1} \mid f^{n+1} \subset C_{i, j, k}\right\}} \Delta U_{i, j, k, f}^{n, n+1} .
$$

As a result, the swept amount verifies the global conservation property

$$
\sum_{C_{i, j, k}} \sum_{\left\{\mathcal{F} \in \mathfrak{F} \mid \mathcal{F}^{n+1} \cap C_{i, j, k} \neq \emptyset\right\}} \Delta U_{i, j, k, \mathcal{F}}^{n, n+1}=\sum_{C_{i, j, k}}\left(\Lambda_{i, j, k}^{n+1}-\Lambda_{i, j, k}^{n}\right) U_{i, j, k}^{n} .
$$

The use of the swept quantity modifies the local conservation at the level of an individual fluid grid cell. Still, the conservation is quasi-local in the sense the exchange of information is made only with the closest neighbours. Finally, we notice that we could have computed directly the integration over the polyhedron $\cup_{t \in\left[t^{n}, t^{n+1}\right]} \mathcal{F} \cap C_{i, j, k}$, without finding a sub-triangular mesh. However, this integration is far more complex computationally than on triangular prisms and the distribution of $\Delta U^{n, n+1}$ over cells would become less accurate.

\subsection{Evaluation of the fluid pressure forces}

In order to ensure the conservation of momentum and energy of the system during the time-step, we need to use the same geometric quantities for the computation of the fluid forces acting on the solid, see (13), and for the solid flux, see (16). We choose here an explicit method which also satisfies consistency properties. The fluid force acting on the solid face $\mathcal{F}$ is evaluated using $\mathcal{F}^{n}$, and we split this face among all the sub-faces contained in $\mathcal{F}^{n}$. This yields $\vec{F}_{\mathcal{F} \text {,fluid }}^{n}=-\vec{\Pi}_{\mathcal{F}}^{n}=\sum_{f \subset \mathcal{F}^{n}} \vec{F}_{f, \text { fluid }}^{n}$, with $\vec{F}_{f \text {,fluid }}^{n}=$ $\left(-\bar{p}_{x}^{n} A_{f}^{n} \nu_{x, f}^{n},-\bar{p}_{y}^{n} A_{f}^{n} \nu_{y, f}^{n},-\bar{p}_{z}^{n} A_{f}^{n} \nu_{z, f}^{n}\right)^{t}:=-\vec{\Pi}_{f}^{n}$. The reason for the computation of the $\bar{p}_{x}^{n}, \bar{p}_{y}^{n}, \bar{p}_{z}^{n}$ on $f^{n}$ is the fact that each sub-face $f^{n}$ is contained only in one cell at time $t^{n}$. In the computation of the solid flux $\Phi_{\text {solid }}^{n}$, we also use a spiting among the sub-faces, $\phi_{i, j, k, \mathcal{F}}^{n}=\sum_{\left\{f^{n} \subset \mathcal{F}^{n} \mid f^{n+1} \subset C_{i, j, k}\right\}} \phi_{i, j, k, f}^{n}$, where

$\phi_{i, j, k, f}^{n}=\left(0, \Pi_{x, f}^{n}, \Pi_{y, f}^{n}, \Pi_{z, f}^{n}, \vec{V}_{f}^{n+\frac{1}{2}} \cdot \vec{\Pi}_{f}^{n}\right)^{t}$, and the velocity $\vec{V}_{f}^{n+\frac{1}{2}}$ is evaluated as $\vec{V}_{f}^{n+\frac{1}{2}}=V^{n+\frac{1}{2}}+$ $\vec{\Omega}^{n+\frac{1}{2}} \wedge\left(\vec{X}_{f}^{n}-\vec{X}^{n}\right)$, where $\vec{X}_{f}^{n}$ is the center of mass of the sub-face $f^{n}$ and $\vec{X}^{n}$ the center of mass of the solid. 


\subsection{Properties of the coupling scheme}

\subsubsection{Conservation}

Conservation of mass and balance of momentum and energy hold for periodic boundary conditions and more generally in all the cases where such properties hold at the continuous level (i.e. mass and energy with fixed boundaries, conservation when boundaries are far ...). The proof is stated in Appendix B. The volume of the rigid solid is preserved by construction and we have verified it numerically.

\subsubsection{Quasi-conservation of energy for the rigid solid}

Let $\mathcal{E}_{s}^{n}$ be the solid energy at time $t^{n}$ defined as $\mathcal{E}_{s}^{n}=\frac{1}{2} m\left\|\vec{V}^{n}\right\|^{2}+\frac{1}{2} \operatorname{tr}\left(\mathbf{P}^{n} \mathbf{D}^{-1}\left(\mathbf{P}^{n}\right)^{t}\right)$. Using Prop. C.1, the expression of $\overrightarrow{\mathcal{M}}_{\text {fluid }}^{n}$ in (17) and the expression of $\vec{V}_{\mathcal{F}}^{n+\frac{1}{2}}$ in (15), the variation of the solid energy over a time step in terms of the fluid forces and torques and of the velocity of the solid is

$$
\begin{aligned}
\mathcal{E}_{s}^{n+1}=\mathcal{E}_{s}^{n}+\Delta t \sum_{\mathcal{F} \in \mathfrak{F}} \vec{F}_{\mathcal{F}, \text { fluid }} \cdot \vec{V}_{\mathcal{F}}^{n+\frac{1}{2}}+\frac{\Delta t^{2}}{8} \operatorname{tr}\left(\mathbf{\Upsilon}^{n} \mathbf{Q}^{n} \mathbf{D}^{-1}\left(\mathbf{Q}^{n}\right)^{t} \mathbf{\Upsilon}^{n}\right) \\
-\frac{\Delta t^{2}}{8} \operatorname{tr}\left(\tilde{\mathbf{\Upsilon}}^{n+1} \mathbf{Q}^{n+1} \mathbf{D}^{-1}\left(\mathbf{Q}^{n+1}\right)^{t} \tilde{\mathbf{\Upsilon}}^{n+1}\right)+\frac{\Delta t^{2}}{32} \operatorname{tr}\left(\mathbf{j}\left(\overrightarrow{\mathcal{M}}^{n}\right) \mathbf{Q}^{n} \mathbf{D}^{-1}\left(\mathbf{Q}^{n}\right)^{t} \mathbf{j}\left(\overrightarrow{\mathcal{M}}^{n}\right)\right) \\
-\frac{\Delta t^{2}}{32} \operatorname{tr}\left(\mathbf{j}\left(\overrightarrow{\mathcal{M}}^{n}\right) \mathbf{Q}^{n+1} \mathbf{D}^{-1}\left(\mathbf{Q}^{n+1}\right)^{t} \mathbf{j}\left(\overrightarrow{\mathcal{M}}^{n}\right)\right)
\end{aligned}
$$

The works of fluid forces and torques cancel with their discrete counterpart in the fluid integration scheme. Concerning the additional four terms, in the two-dimensional case, they vanish due to the commutation of the rotation matrices and their commutation with the matrices $\mathbf{D}$ and $\mathbf{j}(\mathcal{M})$. In the three-dimensional case, the conservation of energy is not exact anymore. However, the discrepancy is limited: the Lagrange multipliers $\boldsymbol{\Upsilon}^{n}$ and $\tilde{\Upsilon}^{n+1}$ are close enough to one another if the rotation matrices $\mathbf{Q}^{n}$ and $\mathbf{Q}^{n+1}$ are close. This provides a second-order error on the energy in terms of the time-step, which we have checked in practice. Therefore, we obtain a quasi-conservation of energy in that case. The proof is stated in Appendix C.

\section{Geometric algorithms for the coupling scheme}

In this section, we present the geometric algorithms required for the implementation of the coupling scheme. We first describe the algorithms used for the detection of the cut-cells as well as the computation of the required informations for each of them. Afterwards, the algorithm used for the evaluation of the swept amount due to the movement of the solid faces during a time step is presented.

\subsection{Cut-cell volume}

At each time step, intersections between the solid boundary and the fluid grid cells need to be computed. The Immersed Boundary method uses various geometric quantities (the volume occupied by the solid in the cut-cell, the occupation of the cut-cell faces, and the boundary areas, see Fig. 1) generated by these intersections. The fluid is discretized and solved on a Cartesian grid. If the solid is not convex, we decompose it into a finite number of convex polyhedral particles. The algorithm introduced here operates on individual fluid grid cells, one at a time. The first part identifies the cut-cells, and the second part computes the polyhedron resulting from the intersection between the fluid grid cell $\mathrm{C}$ and each particle $\mathrm{P}$ composing the solid S.

We distinguish intersection tests which do not construct any intersection objects and intersection algorithms which construct the intersection objects. The first type of algorithm tests if the objects intersect and is fast as it stops after the first encountered intersection. The second type of algorithm constructs all intersection objects. Checking for intersection is easier and much faster than actually computing the intersection result. The algorithm hinges on two major tasks:

- Do convex 3d polyhedra $\mathbf{P}$ and $\mathbf{Q}$ intersect?

For the intersection detection tests, a classical efficient algorithm consists in approximating the geometric primitives (the polyhedra $\mathrm{P}$ and $\mathrm{Q}$ ) with their axis-aligned bounding boxes. If the bounding boxes do 
not intersect, then the objects do not either. Only when a pair of boxes intersect, the exact answer is tested on the complex geometric primitives contained in the boxes.

- Given intersecting convex 3d polyhedra $\mathbf{P}$ and $Q$, compute their intersection.

The polyhedra being convex, we triangulate all the faces of $\mathrm{P}$ and $\mathrm{Q}$ and compute the intersection between the triangular faces from $\mathrm{P}$ and the triangular faces from $\mathrm{Q}$. Thus the problem can be reduced to the computation of the intersection between triangles in three-dimensional space. The intersection can be empty, a point, a segment, a triangle, or a polygon. Finally, the polyhedron resulting from the intersection of $\mathrm{P}$ and $\mathrm{Q}$ is obtained by the computation of the convex hull of all the end points of the segments, triangles and polygons resulting from the intersection between all the triangular faces of $\mathrm{P}$ and Q.

The algorithm for computing the intersection between a fluid grid cell $\mathrm{C}$ and a convex solid particle $\mathrm{P}$ is described in Algorithm 1. All the three-dimensional geometric tasks are handled by CGAL (Computational Geometry Algorithms Library) which is an open source $\mathrm{C}++$ library that contains primitives, data structures and algorithms for computational geometry, as well as a comprehensive documentation [1]. The time required for the determination of the intersections between the solid and the Cartesian fluid

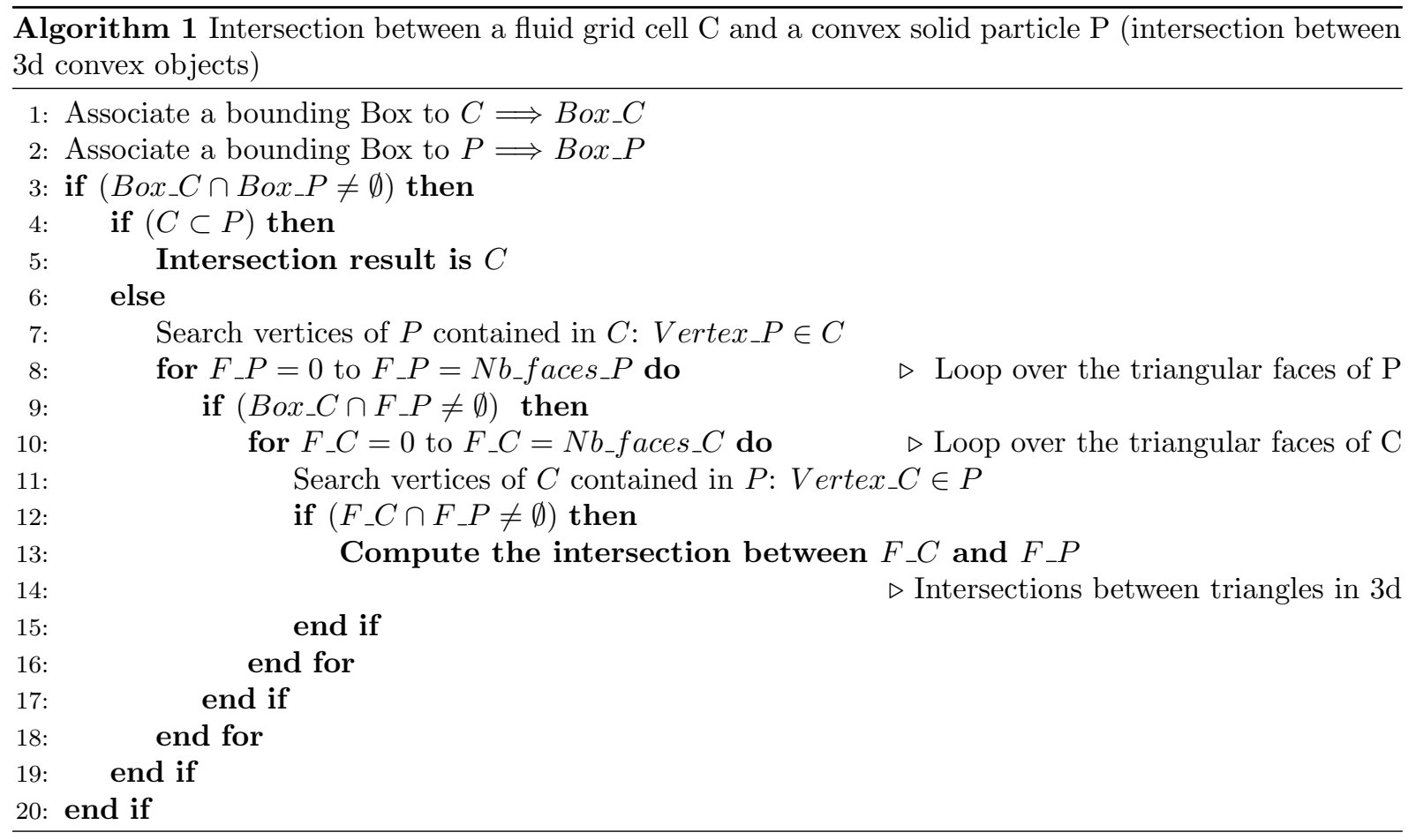

grid and for the computation of the volume of the resulting cut-cells, the occupation of the cut-cells faces and the boundary areas, is comparable to the CPU time required for a fluid flux calculation on one time step for coarse meshes such as the present test cases. However, we have observed that the cost of the intersections increases as $N^{\frac{2}{3}}, N$ being the number of fluid grid cells, as compared to $N$ for the fluid flux so that the overhead is tempered for fine fluid meshes. As the cut-cell volume calculation is performed for each cut-cell separately, the CPU time required for the volume calculation routine scales linearly with the number of cut-cells and the number of the triangles describing the surface of the solid.

\subsection{Swept amount}

For the computation of the amount swept by the movement of the solid face during a time step, we use the following algorithm which we decompose into two major steps. The first step consists in decomposing the solid faces into triangular sub-faces entirely contained in a cell at the discrete times $n$ and $n+1$ (not necessarily the same). The detailed procedure is described in $\S 3.3$. The second step consists in calculating the amount swept by the movement of the triangular sub-face between the discrete times $n$ and $n+1$.

The amount swept by the movement of a triangular sub-face over the time step is the integral of $U^{n}$ over the prism $K_{f}$ whose bases are the triangular sub-faces at the discrete times $n$ and $n+1$, denoted by 
$T^{n}$ and $T^{n+1}$ respectively. The lateral faces of the prism are not necessarily planar. In the case where the prism is entirely contained in one cell we can calculate its signed volume by using the following formula for a prism $P\left(A_{1} B_{1} C_{1}, A_{2} B_{2} C_{2}\right)$ whose bases are the triangles $T\left(A_{1} B_{1} C_{1}\right)$ and $T\left(A_{2} B_{2} C_{2}\right)$ :

$$
\begin{aligned}
\operatorname{Vol}(P)= & \frac{1}{36}\left(2 \overrightarrow{A_{1} B_{1}} \wedge \overrightarrow{A_{1} C_{1}}+2 \overrightarrow{A_{2} B_{2}} \wedge \overrightarrow{A_{2} C_{2}}+\overrightarrow{A_{1} B_{1}} \wedge \overrightarrow{A_{2} C_{2}}+\overrightarrow{A_{2} B_{2}} \wedge \overrightarrow{A_{1} C_{1}}\right) . \\
& \left(\overrightarrow{A_{1} A_{2}}+\overrightarrow{B_{1} B_{2}}+\overrightarrow{C_{1} C_{2}}\right)
\end{aligned}
$$

Otherwise, since $U^{n}$ is piecewise constant, the integral of $U^{n}$ over the prism is computed by first determining the intersection between the prism and the Cartesian fluid grid. Due to the CFL condition, at most eight fluid grid cells intersect the prism $K_{f}$. In order to compute these intersections, we triangulate the lateral faces of the prism with respect to the barycenter of the end-points, and we decompose the prism into tetrahedra (see Fig. 10). Supposing that the prism bases are the triangles $T\left(A_{1} B_{1} C_{1}\right)$ and $T\left(A_{2} B_{2} C_{2}\right)$, we define the points $(A, B, C)$ as barycenters of the four end points of the possibly non-planar faces: $A=\frac{1}{4}\left(B_{1}+B_{2}+C_{1}+C_{2}\right), B=\frac{1}{4}\left(A_{1}+A_{2}+C_{1}+C_{2}\right)$, and $C=\frac{1}{4}\left(A_{1}+A_{2}+B_{1}+B_{2}\right)$. The tetrahedra composing the prism are: $T\left(A_{1} A_{2} C B\right), T\left(B_{1} B_{2} A C\right), T\left(C_{1} C_{2} B A\right), T\left(A_{1} C C_{1} B\right), T\left(B_{1} A C_{1} C\right)$, $T\left(A C B C_{1}\right), T\left(A B C C_{2}\right), T\left(A B_{2} C_{2} C\right), T\left(A_{1} B_{1} C_{1} C\right), T\left(A_{2} C_{2} C B\right)$, and $T\left(A_{2} B_{2} C C_{2}\right)$. Finally, we compute the intersections of these tetrahedra with the fluid grid cells. In particular, we reduce the computation of the intersection between the prism and the Cartesian fluid grid, by proceeding as in $\S 4.1$, to the computation of intersection between triangles in tree-dimension by considering the intersection between the faces of tetrahedra and the triangulated cell faces.

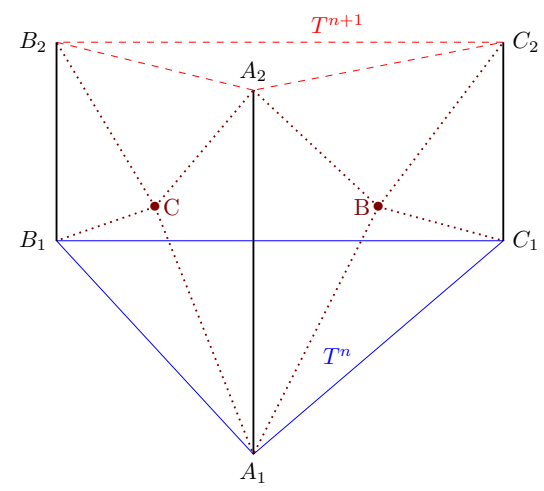

Figure 10: Cutting the "prism" whose bases are $T^{n}$ (continuous line) and $T^{n+1}$ (dashed line) into tetrahedra.

\section{$5 \quad$ Numerical results}

In this section we present numerical results. We first verify the conservation properties of the scheme. Then, we consider the interaction of a shock wave with comparison to $2 \mathrm{D}$ results and with a sphere. We then examine the interaction of doors with a shock wave.

\subsection{Conservation of mass and energy}

In order to verify the conservation of mass and energy by the coupling scheme, we consider a test case consisting of a simple shock tube in a straight rectangular channel and a rigid mobile solid inside this channel. The computational domain is the rectangular box $[0,2] \times[0,1] \times[0,1] \mathrm{m}$ and the initial flow field is given by $\left(\rho=1.4 \mathrm{~kg} \cdot \mathrm{m}^{-3}, p=5 P a, \vec{u}=\overrightarrow{0} \mathrm{~m} \cdot \mathrm{s}^{-1}\right)$, when $x<0.16 \mathrm{~m}$, and by $\left(\rho=1.4 \mathrm{~kg} \cdot \mathrm{m}^{-3}, p=1 \mathrm{~Pa}, \vec{u}=\overrightarrow{0} \mathrm{~m} . \mathrm{s}^{-1}\right)$ otherwise. The initial position of the solid corresponds to the cuboid $(x, y, z) \in[0.4,0.9] \times[0.4,0.6] \times[0.4,0.6] \mathrm{m}$. The computation is performed on a $(140 \times 70 \times 70)$ grid with periodic boundary conditions. The simulation time is $t=1 \mathrm{~s}$.

The pressure and density distribution along the line $\{y=0.5 \mathrm{~m}, z=0.75 \mathrm{~m}\}$ are shown in Fig. 11 . We observe that the shocks and rarefaction waves are well captured, without spurious oscillations. The resolution of the shocks is obviously moderate due to the relative coarseness of the fluid grid. In Fig. 12a 

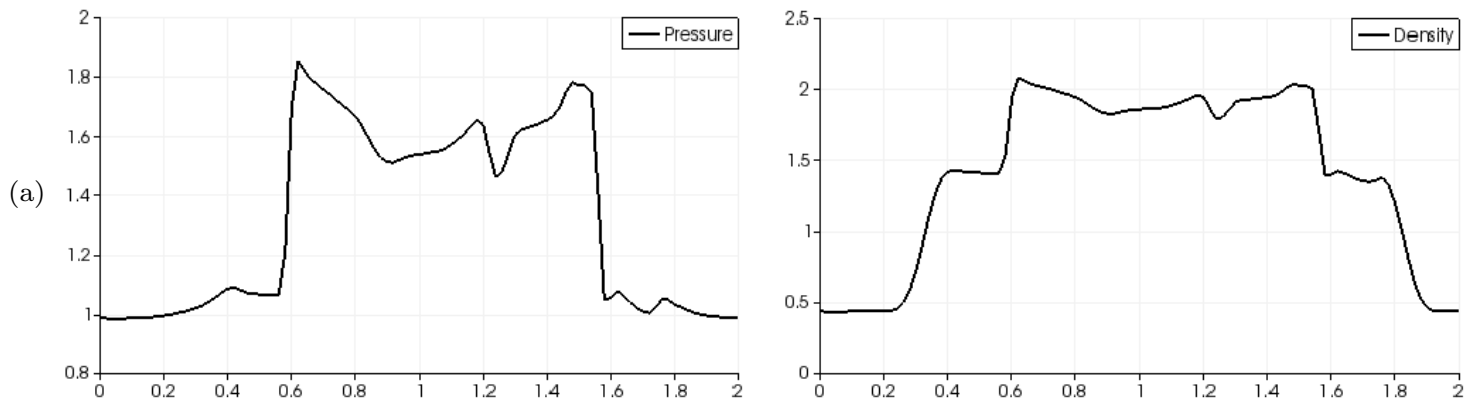

Figure 11: Pressure distribution (a) and density distribution (b) along the line $\{y=0.5 \mathrm{~m}, z=0.75 \mathrm{~m}\}$ at time $t=1 \mathrm{~s}$.

we present the relative conservation error of fluid mass, computed from the difference between the initial total mass and the total mass computed at the different time steps. This mass difference is normalized by the maximum amount of mass swept by the movement of the solid. In Fig. 12b we present the relative energy conservation error, computed as the difference between the initial energy and the energy computed at the different time steps. This energy difference is normalized by the maximum energy exchange between the fluid and the solid, which is the relevant quantity to evaluate the relative effect of coupling on energy conservation. We observe a small variation of both mass and energy, without any clear growth or decrease of either quantity. The variation of mass is as low as $0.01 \%$ of the mass swept by the solid and the variation of energy is as low as $0.01 \%$ of the energy exchange in the system. The main effect accounting for these variations are the rounding errors involved in the evaluation of geometric quantities in cut-cells, since both mass and energy are impacted at similar levels.

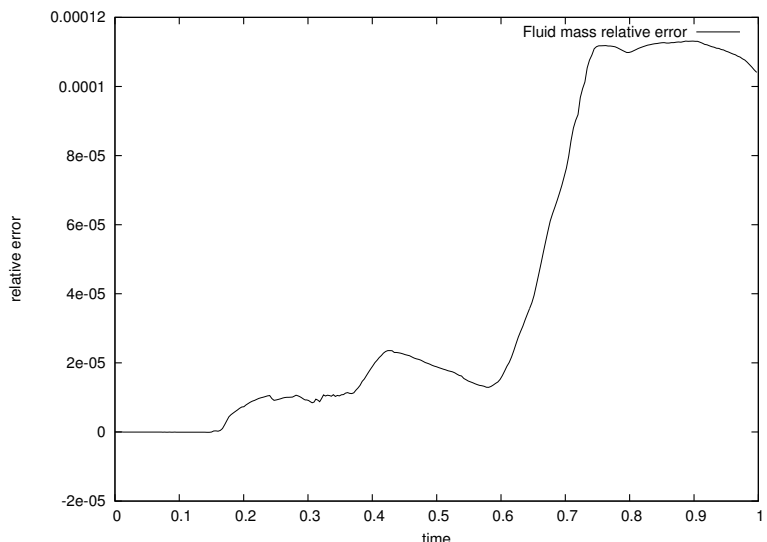

(a)

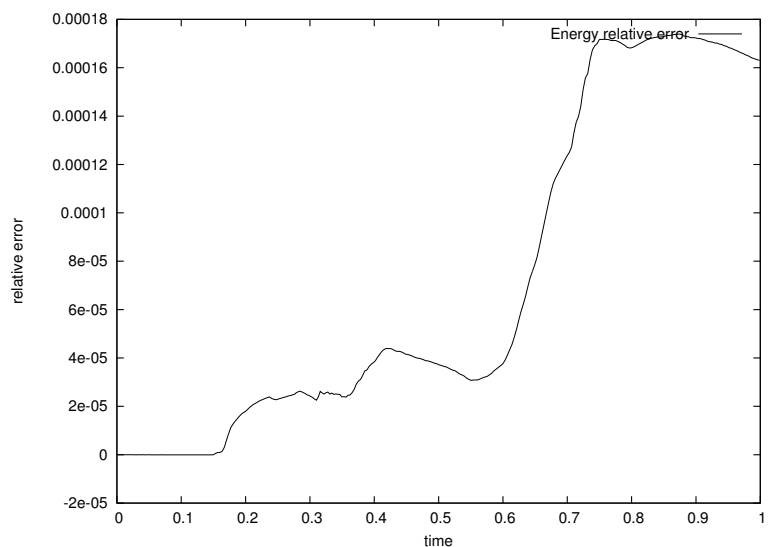

(b)

Figure 12: Relative conservation error on (a) fluid mass and (b) system energy.

\subsection{Interaction of a shock wave and a cylinder}

This moving body test case was first proposed in two space dimensions in [11] using a conservative method and has been studied both with conservative [16, 20] and nonconservative methods [4, 26]. We treat it here in three space dimensions, the third coordinate being degenerate.

A planar shock interacts with a rigid mobile cylinder of density $7.6 \mathrm{~kg} . \mathrm{m}^{-3}$ in a channel. The side boundaries of the domain are rigid walls while the left and right boundaries are respectively inflow and outflow boundaries. The computational domain is the parallelepiped box $[0,1] \times[0,0.2] \times[0,0.2] \mathrm{m}$. The 
shock is initially set up to a Mach number of 3 , so that the initial values are

$$
\begin{cases}\rho=3.85 \mathrm{~kg} \cdot \mathrm{m}^{-3}, p=10.33 \mathrm{~Pa}, u=2.6929 \mathrm{~m} . \mathrm{s}^{-1}, v=w=0 \mathrm{~m} . \mathrm{s}^{-1}, & \text { if } x<0.08 \mathrm{~m}, \\ \rho=1 \mathrm{~kg} \cdot \mathrm{m}^{-3}, p=1 \mathrm{~Pa}, \vec{u}=\overrightarrow{0} \mathrm{~m} . \mathrm{s}^{-1}, & \text { if } x \geq 0.08 \mathrm{~m} .\end{cases}
$$

The cylinder lies on the lower wall of the channel with its axis along the $z$-axis. The initial position of the center of mass of the cylinder is $(0.15,0.05,0.1) \mathrm{m}$, the radius of the cylinder is $R=0.05 \mathrm{~m}$ and its length is $L=0.2 \mathrm{~m}$. The circular section of the cylinder is approximated by a regular polygon with 50 faces. The computation is performed on a $200 \times 40 \times 40$ grid. We impose inflow and outflow boundary conditions at $x=0 \mathrm{~m}$ and $x=1 \mathrm{~m}$ respectively and mirror boundary conditions on the remaining outer boundaries of the fluid domain. The simulation time is $t=0.255 \mathrm{~s}$. The impinging shock wave impacts

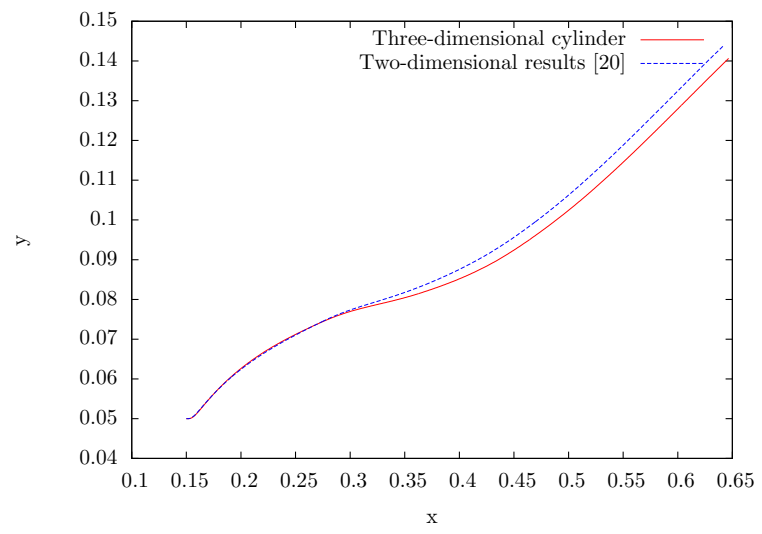

Figure 13: Trajectory of the center of mass of the cylinder in the $(x, y)$-plane.

the cylinder and is partially reflected, while part of the shock wave moves over the cylinder and part of its energy is transferred as kinetic energy to the cylinder. The reflected shock then reflects on the lower wall $(y=0)$, creating an overpressure under the cylinder and lifting it up. Subsequently, a fluid flow develops under the cylinder, resulting in a contact discontinuity which exhibits Kelvin-Helmholtz instabilities. Complex interactions between the cylinder, the walls and the reflected shocks then occur. In Fig. 13 we display the trajectory of the cylinder in the plane $(x, y)$ compared to the two-dimensional trajectory of [20] with the same fluid discretization. The final position of the center of mass of the cylinder is $(0.6465,0.1406,0.099994) \mathrm{m}$. In comparison, the two-dimensional results in [20] yield $(0.643,0.144) \mathrm{m}$ with a similar fluid discretization. This discrepancy is related to the small number of faces (50) of the polygon approximating the circular section in the three-dimensional case compared to the 1240 faces used in the two-dimensional case.

The system is symmetric with respect to the plane $z=0.1 \mathrm{~m}$. We note that the final position of the center of mass of the cylinder remains close to $z=0.1 \mathrm{~m}$. In addition, the velocity of the fluid in the $z$ direction remains small and limited to the Kelvin-Helmholtz instability zones where three-dimensional structures occur. Apart from these features, the invariance in the $z$ direction is well preserved. 30 isocontours of density in the plane $z=1 \mathrm{~m}$ at the final time are plotted in Fig. 14. The position of the shocks agrees very well with [20].

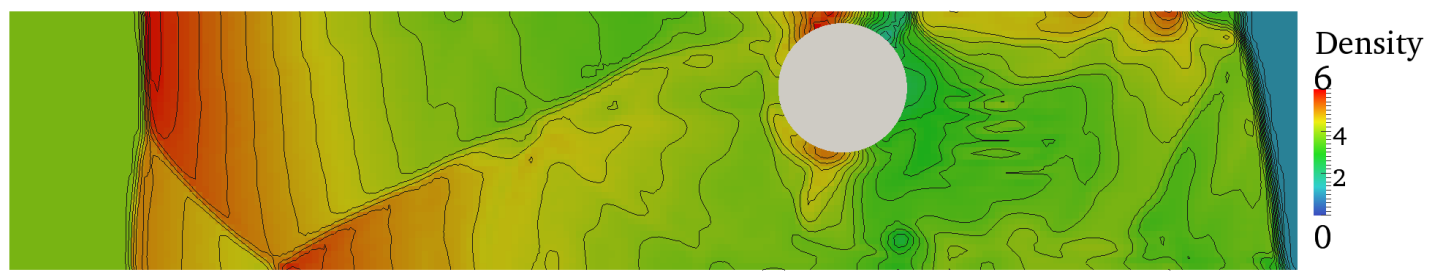

Figure 14: Shock wave/cylinder interaction: 30 iso-contours of density in the plane $z=1 \mathrm{~m}$ at time $t=0.255 \mathrm{~s}$. 


\subsection{Interaction of a shock wave and a sphere}

In this problem, a planar shock interacts with a rigid mobile sphere in a channel. The side boundaries of the domain are rigid walls while the left and right boundaries are respectively inflow and outflow boundaries. The computational domain is the parallelepiped box $[0,1] \times[0,0.2] \times[0,0.2] \mathrm{m}$. The shock is initially set up to a Mach number of 3 , so that the initial values are

$$
\begin{cases}\rho=3.85 \mathrm{~kg} \cdot \mathrm{m}^{-3}, p=10.33 \mathrm{~Pa}, u=2.6929 \mathrm{~m} . \mathrm{s}^{-1}, v=w=0 \mathrm{~m} . \mathrm{s}^{-1}, & \text { if } x<0.08 \mathrm{~m}, \\ \rho=1 \mathrm{~kg} . \mathrm{m}^{-3}, p=1 \mathrm{~Pa}, \vec{u}=\overrightarrow{0} \mathrm{~m} . \mathrm{s}^{-1}, & \text { if } x \geq 0.08 \mathrm{~m} .\end{cases}
$$

The initial position of the center of mass of the sphere is $(0.15,0.05,0.1) \mathrm{m}$, and the radius of the sphere is $R=0.05 \mathrm{~m}$. The sphere is approximated by a polyhedron discretized with 236 faces. The computation is performed on a $400 \times 80 \times 80$ grid. We impose inflow and outflow boundary conditions at $x=0 \mathrm{~m}$ and $x=1 \mathrm{~m}$ respectively and mirror boundary conditions on the remaining outer boundaries of the fluid domain. The simulation time is $t=0.255 \mathrm{~s}$. In the same way that the cylinder interacted with

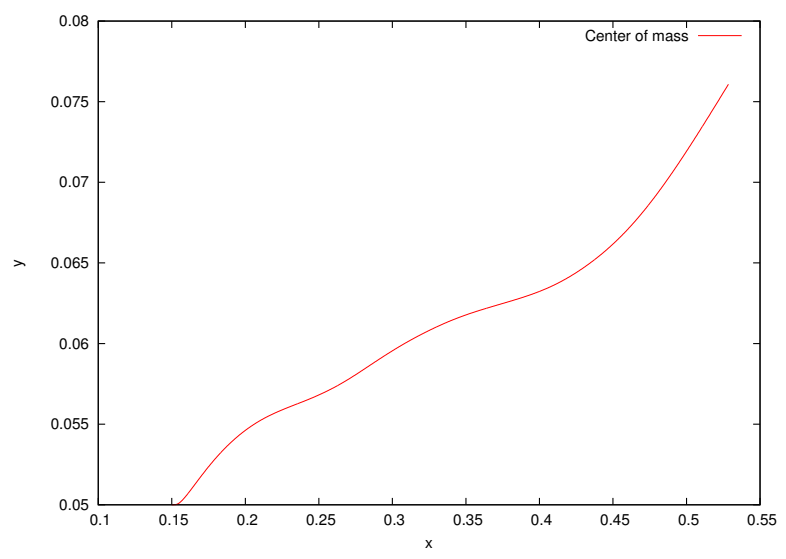

Figure 15: Trajectory of the center of mass of the sphere in the $(x, y)$-plane.

the shock wave in the previous test case $(\S 5.2)$, complex interactions between the sphere, the walls and the reflected shocks occur, creating an overpressure under the sphere and lifting it up. In Fig. 15 we display the trajectory of the sphere in the plane $(x, y)$. The final position of the center of mass of the sphere is $(0.529 \mathrm{~m}, 0.0776 \mathrm{~m}, 0.0984 \mathrm{~m})$. The physical system is symmetric with regard to the plane $z=0.1 \mathrm{~m}$. This feature is fairly well preserved by the numerical results, even though the polyhedron itself is not perfectly symmetric. As a result, the sphere mass center is no longer exactly at $z=0.1 \mathrm{~m}$ at $t=0.255 \mathrm{~s} .30$ iso-contours of density at the final time are plotted in Fig. 16. This computation shows the ability of the coupling algorithm to compute the interaction of strong discontinuities with irregular moving boundaries.

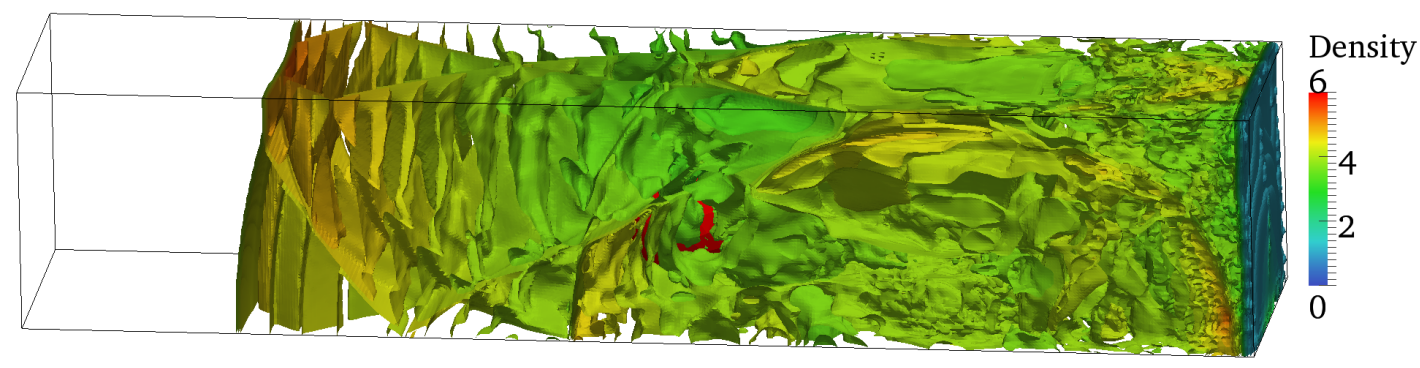

Figure 16: Shock wave/sphere interaction: 30 iso-contours of density at time $t=0.255 \mathrm{~s}$.

\subsection{Interaction of a shock wave with rotating doors}

This case is a three-dimensional analogue of the two-dimensional case presented in [20]. It demonstrates the ability of the method to deal with separating or closing solid boundaries and fluid cells 
including several moving boundaries. These features are of foremost importance in view of being able to deal with fracturing solids.

Four doors initially close a canal and are impacted from the left by a Mach 3 shock. The canal is bounded by four fixed rigid walls on the sides while the two ends along the $x$ axis have inflow and outflow boundary conditions. The fluid domain is the parallelepiped box $[0,2] \times[0,0.5] \times[0,0.5] \mathrm{m}$ and is discretized using a $200 \times 50 \times 50$ grid. The shock is initialized as follows:

$$
\begin{cases}\rho=3.85 \mathrm{~kg} \cdot \mathrm{m}^{-3}, p=10.33 \mathrm{~Pa}, u=2.6929 \mathrm{~m} \cdot \mathrm{s}^{-1}, v=w=0 \mathrm{~m} . \mathrm{s}^{-1}, & \text { if } x<0.43 \mathrm{~m}, \\ \rho=1 \mathrm{~kg} \cdot \mathrm{m}^{-3}, p=1 \mathrm{~Pa}, \vec{u}=\overrightarrow{0} \mathrm{~m} \cdot \mathrm{s}^{-1}, & \text { if } x \geq 0.43 \mathrm{~m} .\end{cases}
$$

The doors are four prisms based on rectangle isoceles triangles, completed on their boundaries by half cylinders. They are presented in Fig. 17(a). Each of them rotates freely around a rotation axis aligned with the axis of the half cylinder on the hypotenuse of the rectangle triangle while its other degrees of freedom are fixed. The diameter of the cylinders is equal to the width of the doors and is $0.05 \mathrm{~m}$. The density of the doors is $0.5 \mathrm{~kg} . \mathrm{m}^{-3}$. The doors entirely block the canal initially.

After the incident shock hits the doors, it reflects to the left and the doors open due to the increase in pressure. The rotation of one of the doors is presented in Fig. 17(b). Due to the symmetry of the problem, the rotation of each door should be the same, and we have verified that the solution is almost symmetric. We observe that the doors are rotated all the way to 90 degrees, at which point they stop, having removed the fluid from the cells next to the boundary wall. In Fig. 18 and 19, we present the evolution of the fluid density field in planes $z=0.25 \mathrm{~m}$ and $y=z$, respectively, at times $0.02 \mathrm{~s}, 0.05 \mathrm{~s}$, $0.1 \mathrm{~s}$ and $0.25 \mathrm{~s}$. In order to help visualize the fluid flow, we have removed the particule with rotation axis $(x=0.5 \mathrm{~m}, z=0.45 \mathrm{~m})$ in Fig. 18. The opening of the doors results in compression waves being created by the movement of the doors, while the pressure and density decrease in the center of the canal. Complex interactions of waves occur due to door movements and interaction with walls. Once the doors are rotated at 90 degrees, the fluid evolution is similar to a nozzle flow due to the static presence of the doors. We note the fact that the symmetry of the flow about the planes of symmetry of the canal is very well preserved by the coupling method.

(a)

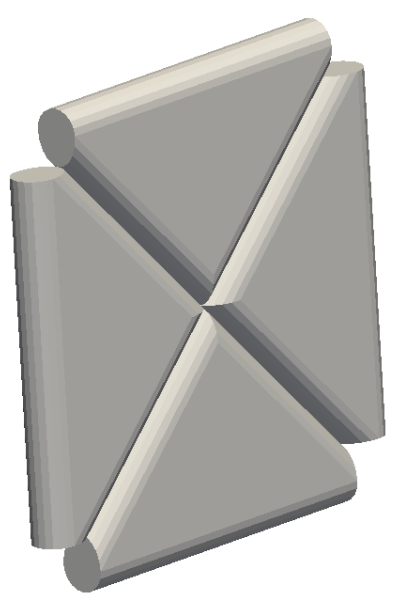

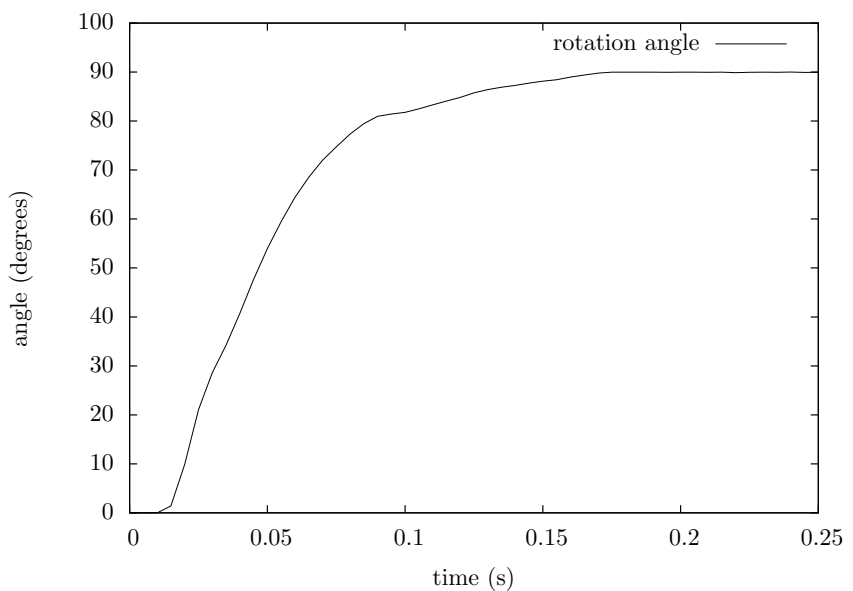

(b)

Figure 17: Initial position of the doors (a), evolution of the rotation of the doors in time (b).

\section{Conclusion}

We have developed a coupling method between an inviscid compressible fluid and a three-dimensional moving rigid solid, extending the explicit coupling scheme with a two-dimensional rigid solid of [20]. The extension has been achieved through exact geometric intersections of the solid boundary and the fluid grid. The method yields exact conservation of mass, momentum and energy of the system, and also exhibits important consistency properties, such as conservation of uniform movement of both fluid and solid as well as the absence of numerical roughness on a straight boundary.

The computational cost of the fluid and solid methods essentially results from the evaluation of fluxes on the fluid side and of forces and torques on the solid side. We emphasize that the coupling algorithm 


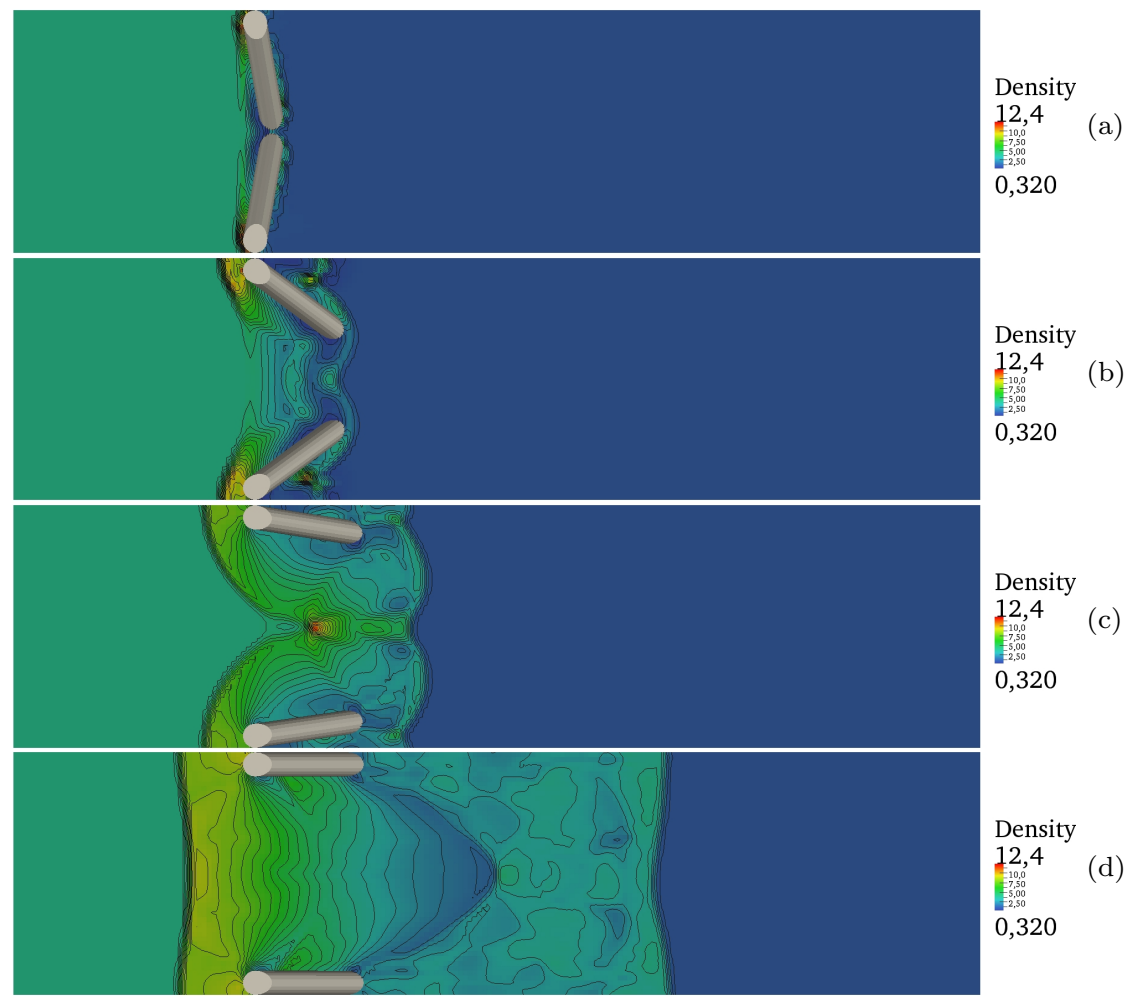

Figure 18: Shock wave/doors interaction: 30 iso-contours of density in the plane $z=0.25 \mathrm{~m}$ at times $t=0.02 \mathrm{~s}$ (a), $t=0.05 \mathrm{~s} \mathrm{(b),} t=0.1 \mathrm{~s} \mathrm{(c)} \mathrm{and} t=0.25 \mathrm{~s}(\mathrm{~d})$.

evaluates these only once per time step, ensuring computational efficiency. Regarding surface coupling, the algorithm overhead scales as the number of solid faces and as $N^{\frac{2}{3}}, N$ being the number of fluid grid cells. In comparison, the fluid flux computation time scales as $N$.

The presented test cases allowed us to verify the main properties of the coupling scheme and to illustrate the ability of the method to compute the interaction of strong discontinuities with rigid solids undergoing large displacement. The next step is to move on to more complex test cases and to enrich the algorithm to take into account the deformation and the fracture of the solid. The algorithm has been designed in order to facilitate the extension of these results to solid deformation and fracture. This would require the discretization of the solid body using particles in the context of the DEM, an adequate reconstruction of the solid boundary resulting from the relative movement between the particles composing the solid, an appropriate procedure to fill the ghost-cells and the definition of a map (not necessarily bijective due to the opening of the fracture in one point) providing the correspondence from the position of the boundary at time $t^{n}$ to its position at time $t^{n+1}$, see $[24,25]$.

Acknowledgement This work was supported in part by CEA/DAM. The authors are grateful to Virginie Daru (LIMSI, CNRS), Alexandre Ern (CERMICS, Université Paris-Est), Christian Mariotti (CEA/DAM) and Christian Tenaud (LIMSI, CNRS) for stimulating discussions.

\section{A Flux on mobile boundary}

In this section, we present the Finite Volume discretization of a conservative law in the case when the boundaries of the cell are mobile, as considered in [9]. This discretization is used in the Immersed Boundary method in cut-cells, see 3.1. For simplicity, we consider the one-dimensional case. The Euler equations (1) are given by

$$
\frac{\partial U}{\partial t}+\frac{\partial F(U)}{\partial x}=0
$$




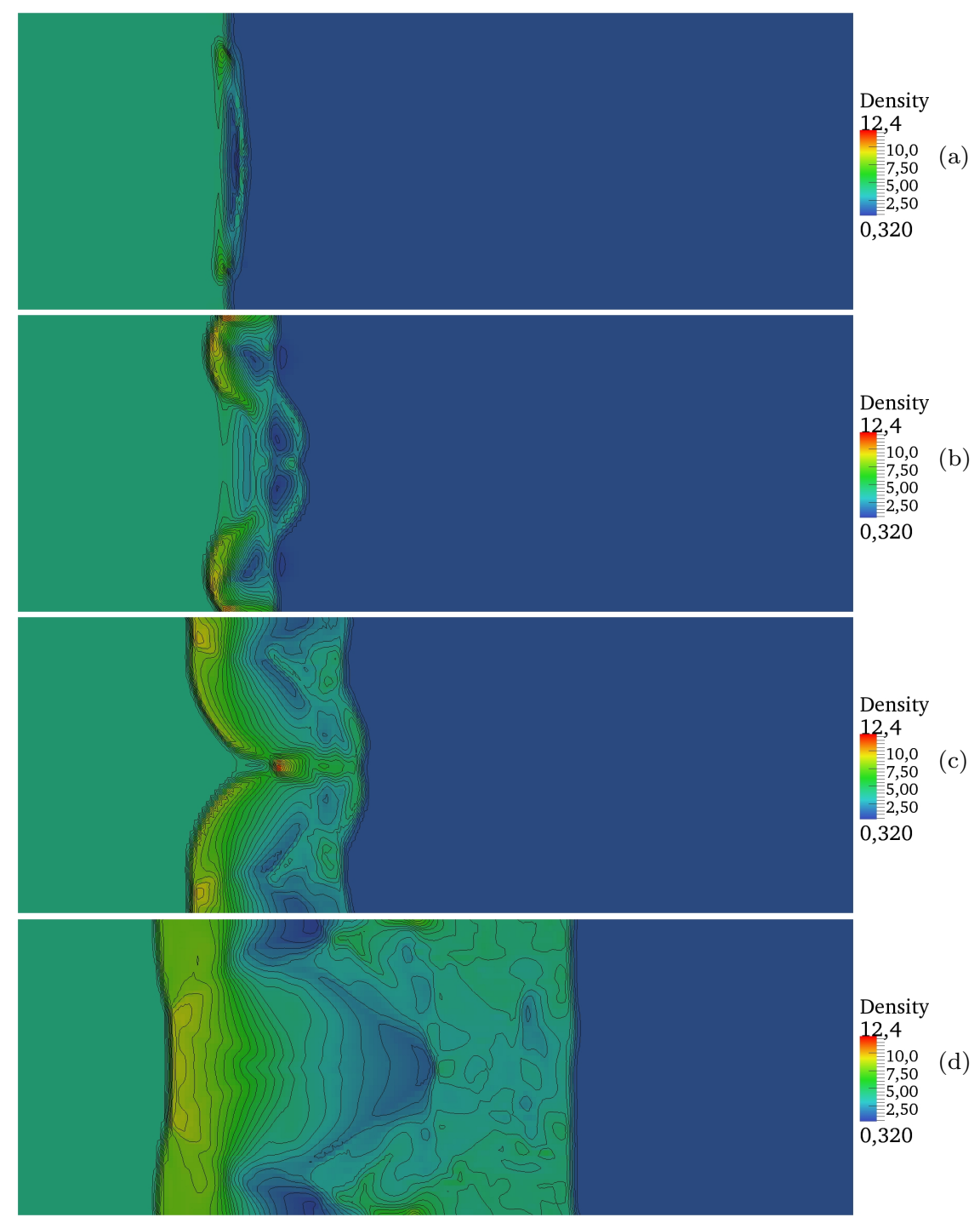

Figure 19: Shock wave/doors interaction: 30 iso-contours of density in the plane $y=z$ at times $t=0.02 \mathrm{~s}$ (a), $t=0.05 \mathrm{~s}(\mathrm{~b}), t=0.1 \mathrm{~s} \mathrm{(c)} \mathrm{and} t=0.25 \mathrm{~s}(\mathrm{~d})$.

where the vector of conservative variables $U$ is given by $U=(\rho, \rho u, \rho E)^{t}$ and the flux function is given by $F(U)=\left(\rho u, \rho u^{2}+p,(\rho E+p) u\right)^{t}$.

We consider a grid in the plane $(x, t)$ defined by the points $x_{i+1 / 2}$ and the time step $\Delta t$ which is supposed to be constant for simplicity. The space step is denoted by $\Delta x_{i}=x_{i+1 / 2}-x_{i-1 / 2}$. We denote by $U_{i}^{n}$ an approximation of the average value of $U$ in the cell $C_{i}=\left[x_{i-1 / 2}, x_{i+1 / 2}\right]$ at time $t^{n}$.

As described in Section 3, the solid is superimposed to the fluid grid. Thus, the cell $\left[x_{i-1 / 2}, x_{i+1 / 2}\right]$ can be partially covered by the solid. Let us consider the time-space cell $B_{i}^{n+1 / 2}$ illustrated in Fig. 20, where we consider that the point $\chi_{i-1 / 2}$ is fixed in time at the point $x_{i-1 / 2}$ and the point $\chi_{i+1 / 2}$ varies in time due to the presence of a solid boundary in the cell. We denote by $B_{i}^{n}=\left[\chi_{i-1 / 2}^{n}, \chi_{i+1 / 2}^{n}\right]$ the cell at time $t^{n}$, and by $B_{i}^{n+1}=\left[\chi_{i-1 / 2}^{n+1}, \chi_{i+1 / 2}^{n+1}\right]$ the cell at time $t^{n+1}$. We integrate the conservation law (20) in the timespace cell $B_{i}^{n+1 / 2}$, and by using the divergence formula, we obtain $\int_{\partial B_{i}^{n+1 / 2}}\left(U n_{t}+F(U(t, x)) n_{x}\right) d \gamma=0$, where the outward normal $\vec{n}$ on $\partial B_{i}^{n+1 / 2}$ has the form $\vec{n}=\left(n_{t}, n_{x}\right)$. Taking into account the notation of 


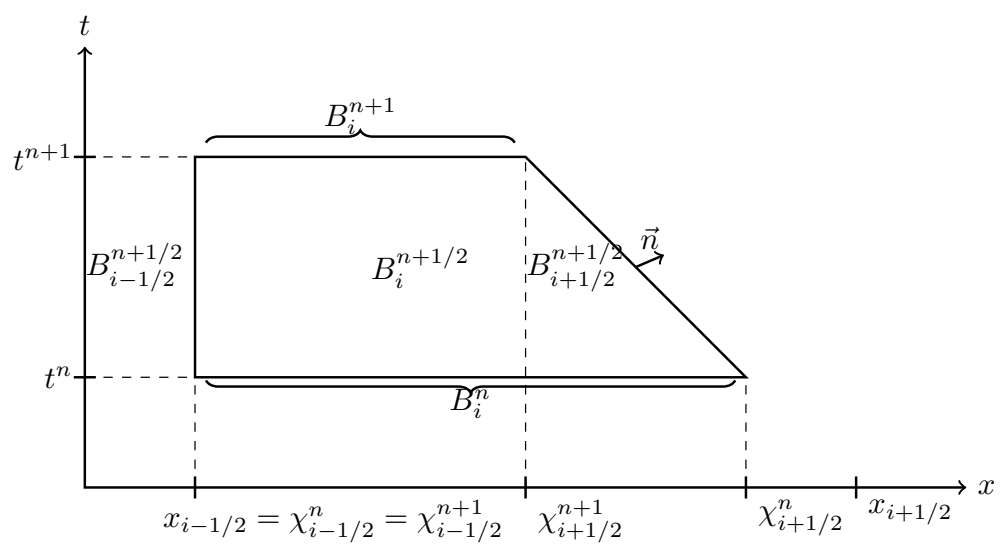

Figure 20: Time-space cell

Fig. 20, we infer

$$
\begin{aligned}
\int_{\partial B_{i}^{n+1 / 2}} & \left(U n_{t}+f(U(t, x)) n_{x}\right) d \gamma=\int_{B_{i}^{n+1}} U n_{t} d \gamma+\int_{B_{i}^{n}} U n_{t} d \gamma \\
& +\int_{B_{i-1 / 2}^{n+1 / 2}}\left(U n_{t}+F(U(t, x)) n_{x}\right) d \gamma+\int_{B_{i+1 / 2}^{n+1 / 2}}\left(U n_{t}+F(U(t, x)) n_{x}\right) d \gamma
\end{aligned}
$$

The first two terms are evaluated as $\int_{B_{i}^{n+1}} U n_{t} d \gamma=\left|B_{i}^{n+1}\right| U_{i}^{n+1}=\left(\Delta x_{i}-\left(x_{i+1 / 2}-\chi_{i+1 / 2}^{n+1}\right)\right) U_{i}^{n+1}$ and $\int_{B_{i}^{n}} U n_{t} d \gamma=-\left|B_{i}^{n}\right| U_{i}^{n}=-\left(\Delta x_{i}-\left(x_{i+1 / 2}-\chi_{i+1 / 2}^{n}\right)\right) U_{i}^{n}$. We denote by $\Lambda_{i}^{n+1}$ and $\Lambda_{i}^{n}$ the volume fractions occupied by the solid in the cell at times $t^{n+1}$ and $t^{n}$, respectively given by: $\Lambda_{i}^{n+1}=$ $\frac{x_{i+1 / 2}-\chi_{i+1 / 2}^{n+1}}{\Delta x_{i}}$ and $\Lambda_{i}^{n}=\frac{x_{i+1 / 2}-\chi_{i+1 / 2}^{n}}{\Delta x_{i}}$. We infer: $\int_{B_{i}^{n+1}} U n_{t} d \gamma=\Delta x_{i}\left(1-\Lambda_{i}^{n+1}\right) U_{i}^{n+1}$ and $\int_{B_{i}^{n}} U n_{t} d \gamma=$ $-\Delta x_{i}\left(1-\Lambda_{i}^{n}\right) U_{i}^{n}$. The flux of $F$ on the boundary $B_{i-1 / 2}^{n+1 / 2}$ can be approximated by the usual numerical flux $F_{i-1 / 2}^{n}: \int_{B_{i-1 / 2}^{n}} F(U(t, x)) n_{x} d \gamma \simeq-\Delta t F_{i-1 / 2}^{n+1 / 2}$. The mean velocity of the point $\chi_{i+1 / 2}$ between $t^{n}$ and $t^{n+1}$ is given by $w_{i+1 / 2}^{n+1 / 2}=\frac{1}{\Delta t}\left(\chi_{i+1 / 2}^{n+1}-\chi_{i+1 / 2}^{n}\right)$. The mobile boundary segment $B_{i+1 / 2}^{n+1 / 2}$ has a normal direction $\vec{n}$ such that $n_{x}=\frac{1}{\sqrt{1+\left(w_{i+1 / 2}^{n+1 / 2}\right)^{2}}}$ and $n_{t}=-\frac{w_{i+1 / 2}^{n+1 / 2}}{\sqrt{1+\left(w_{i+1 / 2}^{n+1 / 2}\right)^{2}}}$. Thus, we obtain $\int_{B_{i+1 / 2}^{n+1 / 2}}\left(U n_{t}+f(U(t, x)) n_{x}\right) d \gamma=$ $\int_{t^{n}}^{t^{n+1}}\left(-w_{i+1 / 2}^{n+1 / 2} U\left(t, x_{i+1 / 2}(t)\right)+F\left(U\left(t, x_{i+1 / 2}(t)\right)\right)\right) d t$. Taking into account the particular form of the flux function of $F$ and the fact that the state velocity $U_{i+1 / 2}^{n+1 / 2}$ is exactly equal to $w_{i+1 / 2}^{n+1 / 2}$, we infer

$$
\int_{B_{i+1 / 2}^{n+1 / 2}}\left(U n_{t}+F(U(t, x)) n_{x}\right) d \gamma=\Delta t\left(0, p_{i+1 / 2}^{n+1 / 2}, p_{i+1 / 2}^{n+1 / 2} w_{i+1 / 2}^{n+1 / 2}\right)^{t}
$$

where $p_{i+1 / 2}^{n+1 / 2}$ is the pressure of the state $U_{i+1 / 2}^{n+1 / 2}$. Finally, gathering the four terms we obtain (1$\left.\Lambda_{i}^{n+1}\right) U_{i}^{n+1}=\left(1-\Lambda_{i}^{n}\right) U_{i}^{n}+\Delta t\left(\Phi_{i, \text { fluid }}^{n+1 / 2}+\Phi_{i, \text { solid }}^{n+1 / 2}\right)$,

where $\Phi_{i, \text { fluid }}^{n+1 / 2}=\frac{1}{\Delta x_{i}} F_{i-1 / 2}^{n+1 / 2}$ and $\Phi_{i, \text { solid }}^{n+1 / 2}=\frac{-1}{\Delta x_{i}}\left(0, p_{i+1 / 2}^{n+1 / 2}, p_{i+1 / 2}^{n+1 / 2} w_{i+1 / 2}^{n+1 / 2}\right)^{t}$. 


\section{B Conservation of mass, balance of momentum and energy}

We denote by $V=(\Delta x \Delta y \Delta z)$ the volume of the fluid grid cells. Summing (11) over all the fluid grid cells $C_{i, j, k}$, the cancellation of fluxes on each fluid grid cell face implies

$$
\begin{aligned}
\sum_{C_{i, j, k}}\left(1-\Lambda_{i, j, k}^{n+1}\right) U_{i, j, k}^{n+1}=\sum_{C_{i, j, k}}\left(1-\Lambda_{i, j, k}^{n+1}\right) U_{i, j, k}^{n} & +\frac{\Delta t}{V} \sum_{C_{i, j, k}} \sum_{\left\{\mathcal{F} \in \mathfrak{F} \mid \mathcal{F}^{n+1} \cap C_{i, j, k} \neq \emptyset\right\}} \phi_{i, j, k, \mathcal{F}}^{n} \Delta U_{i, j, k, \mathcal{F}}^{n, n+1} \\
& +\sum_{C_{i, j, k}} \sum_{\left\{\mathcal{F} \in \mathfrak{F} \mid \mathcal{F}^{n+1} \cap C_{i, j, k} \neq \emptyset\right\}}
\end{aligned}
$$

Using (19) yields $\sum_{C_{i, j, k}}\left(1-\Lambda_{i, j, k}^{n+1}\right) U_{i, j, k}^{n+1}=\sum_{C_{i, j, k}}\left(1-\Lambda_{i, j, k}^{n}\right) U_{i, j, k}^{n}+\frac{\Delta t}{V} \sum_{\mathcal{F} \in \mathfrak{F}} \phi_{\mathcal{F}}^{n}$. We finally obtain

$$
\frac{1}{V} \int_{\Omega_{\text {fluid }}^{n+1}} U^{n+1}=\frac{1}{V} \int_{\Omega_{\text {fluid }}^{n}} U^{n}+\frac{\Delta t}{V} \sum_{\mathcal{F} \in \mathfrak{F}} \phi_{\mathcal{F}}^{n} .
$$

The expression of $\phi_{\mathcal{F}}^{n}$ in (16) shows that the first component is equal to zero. Hence, the first component of (21) expresses the fluid mass conservation. Replacing the expression of $\phi_{\mathcal{F}}^{n}$ from (16) in the fluid momentum and energy equations, leads to

$$
\begin{aligned}
\int_{\Omega_{\text {fluid }}^{n+1}} \rho^{n+1} \vec{u}^{n+1} & =\int_{\Omega_{\text {fluid }}^{n}} \rho^{n} \vec{u}^{n}+\Delta t \sum_{\mathcal{F} \in \mathcal{F}} \int_{\mathcal{F}^{n}} \vec{\Pi}_{\mathcal{F}}^{n}, \\
\int_{\Omega_{\text {fluid }}^{n+1}} \rho^{n+1} E^{n+1} & =\int_{\Omega_{\text {fluid }}^{n}} \rho^{n} E^{n}+\Delta t \sum_{\mathcal{F} \in \mathcal{F}} \int_{\mathcal{F}^{n}} \vec{V}_{\mathcal{F}}^{n+\frac{1}{2}} \cdot \vec{\Pi}_{\mathcal{F}}^{n} .
\end{aligned}
$$

The fluid pressure force applied on the solid face $\mathcal{F} \in \mathfrak{F}$ during the time step is given by (13). The solid momentum variation induced by the pressure forces on $\mathcal{F}$, denoted $\Delta P_{\mathcal{F}}$, and the corresponding energy variation, denoted $\Delta E_{\mathcal{F}}$, are given by

$$
\begin{aligned}
& \Delta P_{\mathcal{F}}=\Delta t \vec{F}_{\mathcal{F}, \text { fluid }}^{n}=-\Delta t \int_{\mathcal{F}^{n}} \vec{\Pi}_{\mathcal{F}}^{n}, \\
& \Delta E_{\mathcal{F}}=\Delta t \vec{F}_{\mathcal{F}, \text { fluid }}^{n} \cdot \vec{V}_{\mathcal{F}}^{n+\frac{1}{2}}=-\Delta t \vec{V}_{\mathcal{F}}^{n+\frac{1}{2}} \cdot \int_{\mathcal{F}^{n}} \vec{\Pi}_{\mathcal{F}}^{n} .
\end{aligned}
$$

Thus, the balance of momentum and energy in the fluid domain results in

$$
\begin{aligned}
\int_{\Omega_{\text {fluid }}^{n+1}} \rho^{n+1} \vec{u}^{n+1}+\sum_{\mathcal{F} \in \mathfrak{F}} \Delta P_{\mathcal{F}} & =\int_{\Omega_{\text {fluid }}^{n}} \rho^{n} \vec{u}^{n}, \\
\int_{\Omega_{\text {fluid }}^{n+1}} \rho^{n+1} E^{n+1}+\sum_{\mathcal{F} \in \mathcal{F}} \Delta E_{\mathcal{F}} & =\int_{\Omega_{\text {fluid }}^{n}} \rho^{n} E^{n} .
\end{aligned}
$$

\section{Quasi-conservation of energy for the rigid solid}

Proposition C.1. The variation of the solid energy over a time step in terms of the fluid forces and torques and of the velocity of the solid is

$$
\begin{aligned}
& \mathcal{E}_{s}^{n+1}= \mathcal{E}_{s}^{n}+\Delta t \vec{F}_{\text {fluid }}^{n} \cdot \vec{V}^{n+\frac{1}{2}}+\Delta t \overrightarrow{\mathcal{M}}_{\text {fluid }}^{n} \cdot \vec{\Omega}^{n+\frac{1}{2}}+ \\
&-\frac{\Delta t^{2}}{8} \operatorname{tr}\left(\mathbf{\Upsilon}^{n} \mathbf{Q}^{n} \mathbf{D}^{-1}\left(\mathbf{Q}^{n}\right)^{t} \mathbf{\Upsilon}^{n}\right) \\
& \operatorname{tr}\left(\tilde{\mathbf{\Upsilon}}^{n+1} \mathbf{Q}^{n+1} \mathbf{D}^{-1}\left(\mathbf{Q}^{n+1}\right)^{t} \tilde{\mathbf{\Upsilon}}^{n+1}\right)+ \frac{\Delta t^{2}}{32} \operatorname{tr}\left(\mathbf{j}\left(\overrightarrow{\mathcal{M}}_{\text {fluid }}^{n}\right) \mathbf{Q}^{n} \mathbf{D}^{-1}\left(\mathbf{Q}^{n}\right)^{t} \mathbf{j}\left(\overrightarrow{\mathcal{M}}_{\text {fluid }}^{n}\right)\right) \\
&-\frac{\Delta t^{2}}{32} \operatorname{tr}\left(\mathbf{j}\left(\overrightarrow{\mathcal{M}}_{\text {fluid }}^{n}\right) \mathbf{Q}^{n+1} \mathbf{D}^{-1}\left(\mathbf{Q}^{n+1}\right)^{t} \mathbf{j}\left(\overrightarrow{\mathcal{M}}_{\text {fluid }}^{n}\right)\right) .
\end{aligned}
$$


Proof. Recall that if $\mathbf{A}$ and $\mathbf{S}$ are respectively a skew-symmetric and a symmetric matrix, then

$$
\operatorname{tr}(\mathbf{A S})=0 .
$$

Developing $\mathcal{E}_{s}^{n+1}$ using equations (2)-(5), we obtain

$$
\begin{aligned}
\mathcal{E}_{s}^{n+1} & =\frac{1}{2} m\left\|\vec{V}^{n}\right\|^{2}+\Delta t \vec{F}_{\text {fluid }}^{n} \cdot \vec{V}^{n+\frac{1}{2}}+\frac{1}{2} \operatorname{tr}\left(\mathbf{P}^{n} \mathbf{D}^{-1}\left(\mathbf{P}^{n}\right)^{t}\right)+\frac{\Delta t}{2} \operatorname{tr}\left(\mathbf{P}^{n+1} \mathbf{D}^{-1}\left(\mathbf{Q}^{n+1}\right)^{t} \tilde{\mathbf{\Upsilon}}^{n+1}\right) \\
& +\frac{\Delta t}{2} \operatorname{tr}\left(\mathbf{P}^{n} \mathbf{D}^{-1}\left(\mathbf{Q}^{n}\right)^{t} \mathbf{\Upsilon}^{n}\right)+\frac{\Delta t}{2} \operatorname{tr}\left(\mathbf{j}\left(\overrightarrow{\mathcal{M}}_{\text {fluid }}^{n}\right)\left(\mathbf{Q}^{n}+\mathbf{Q}^{n+1}\right) \mathbf{D}^{-1}\left(\mathbf{P}^{n+\frac{1}{2}}\right)^{t}\right) \\
& +\frac{\Delta t^{2}}{8} \operatorname{tr}\left(\mathbf{\Upsilon}^{n} \mathbf{Q}^{n} \mathbf{D}^{-1}\left(\mathbf{Q}^{n}\right)^{t} \mathbf{\Upsilon}^{n}\right)-\frac{\Delta t^{2}}{8} \operatorname{tr}\left(\tilde{\mathbf{\Upsilon}}^{n+1} \mathbf{Q}^{n+1} \mathbf{D}^{-1}\left(\mathbf{Q}^{n+1}\right)^{t} \tilde{\mathbf{\Upsilon}}^{n+1}\right) \\
& +\frac{\Delta t^{2}}{32} \operatorname{tr}\left(\mathbf{j}\left(\overrightarrow{\mathcal{M}}_{\text {fluid }}^{n}\right) \mathbf{Q}^{n} \mathbf{D}^{-1}\left(\mathbf{Q}^{n}\right)^{t} \mathbf{j}\left(\overrightarrow{\mathcal{M}}_{\text {fluid }}^{n}\right)\right)-\frac{\Delta t^{2}}{32} \operatorname{tr}\left(\mathbf{j}\left(\overrightarrow{\mathcal{M}}_{\text {fluid }}^{n}\right) \mathbf{Q}^{n+1} \mathbf{D}^{-1}\left(\mathbf{Q}^{n+1}\right)^{t} \mathbf{j}\left(\overrightarrow{\mathcal{M}}_{\text {fluid }}^{n}\right)\right) .
\end{aligned}
$$

Using (24), (7) at times $t^{n}$ and $t^{n+1}$ and the symmetry of $\boldsymbol{\Upsilon}^{n}$ and $\tilde{\boldsymbol{\Upsilon}}^{n+1}$, the fourth and fifth term vanish. We now prove the following result to estimate the sixth term on the right-hand side.

Lemma C.2. $\mathbf{P}^{n+\frac{1}{2}} \mathbf{D}^{-1}\left(\mathbf{Q}^{n}+\mathbf{Q}^{n+1}\right)^{t}$ is a skew-symmetric matrix, so that we can define the angular velocity vector $\vec{\Omega}^{n+\frac{1}{2}}$ at time $\left(n+\frac{1}{2}\right) \Delta t$ by

$\mathbf{j}\left(\vec{\Omega}^{n+\frac{1}{2}}\right)=\frac{1}{2} \mathbf{P}^{n+\frac{1}{2}} \mathbf{D}^{-1}\left(\mathbf{Q}^{n}+\mathbf{Q}^{n+1}\right)^{t}$.

Proof. Let us note that $\mathbf{P}^{n+\frac{1}{2}} \mathbf{D}^{-1}=\frac{1}{\Delta t}\left(\mathbf{Q}^{n+1}-\mathbf{Q}^{n}\right)$. It follows that

$$
\mathbf{P}^{n+\frac{1}{2}} \mathbf{D}^{-1}\left(\mathbf{Q}^{n}+\mathbf{Q}^{n+1}\right)^{t}=\frac{1}{\Delta t}\left(\mathbf{Q}^{n+1}-\mathbf{Q}^{n}\right)\left(\mathbf{Q}^{n}+\mathbf{Q}^{n+1}\right)^{t}=\frac{1}{\Delta t}\left(\mathbf{Q}^{n+1}\left(\mathbf{Q}^{n}\right)^{t}-\mathbf{Q}^{n}\left(\mathbf{Q}^{n+1}\right)^{t}\right) .
$$

which proves the result.

We can now finish the proof. It is straightforward to see that $\operatorname{tr}\left(\mathbf{j}\left(\overrightarrow{\mathcal{M}}_{\text {fluid }}^{n}\right) \mathbf{j}\left(\vec{\Omega}^{n+\frac{1}{2}}\right)\right)=-2 \overrightarrow{\mathcal{M}}_{\text {fluid }}^{n} \cdot \vec{\Omega}^{n+\frac{1}{2}}$. Finally, we obtain the variation of the discrete solid energy in terms of the fluid forces and torques and of the velocity of the solid as follows:

$$
\begin{aligned}
\mathcal{E}_{s}^{n+1}=\mathcal{E}_{s}^{n}+\Delta t \vec{F}_{\text {fluid }}^{n} \cdot \vec{V}^{n+\frac{1}{2}}+\Delta t \overrightarrow{\mathcal{M}}_{\text {fluid }}^{n} \cdot \vec{\Omega}^{n+\frac{1}{2}} & +\frac{\Delta t^{2}}{8} \operatorname{tr}\left(\mathbf{\Upsilon}^{n} \mathbf{Q}^{n} \mathbf{D}^{-1}\left(\mathbf{Q}^{n}\right)^{t} \mathbf{\Upsilon}^{n}\right) \\
-\frac{\Delta t^{2}}{8} \operatorname{tr}\left(\tilde{\mathbf{\Upsilon}}^{n+1} \mathbf{Q}^{n+1} \mathbf{D}^{-1}\left(\mathbf{Q}^{n+1}\right)^{t} \tilde{\mathbf{\Upsilon}}^{n+1}\right) & +\frac{\Delta t^{2}}{32} \operatorname{tr}\left(\mathbf{j}\left(\overrightarrow{\mathcal{M}}_{\text {fluid }}^{n}\right) \mathbf{Q}^{n} \mathbf{D}^{-1}\left(\mathbf{Q}^{n}\right)^{t} \mathbf{j}\left(\overrightarrow{\mathcal{M}}_{\text {fluid }}^{n}\right)\right) \\
& -\frac{\Delta t^{2}}{32} \operatorname{tr}\left(\mathbf{j}\left(\overrightarrow{\mathcal{M}}_{\text {fluid }}^{n}\right) \mathbf{Q}^{n+1} \mathbf{D}^{-1}\left(\mathbf{Q}^{n+1}\right)^{t} \mathbf{j}\left(\overrightarrow{\mathcal{M}}_{\text {fluid }}^{n}\right)\right) .
\end{aligned}
$$

\section{REFERENCES}

[1] Computational geometry algorithms library. http://www.cgal.org/.

[2] R. Abgrall and S. Karni. Ghost-fluids for the poor: a single fluid algorithm for multifluids, volume 141 of Internat. Ser. Numer. Math., pages 1-10. Birkhäuser, 2001.

[3] A. Ambroso, C. Chalons, and P.-A. Raviart. A Godunov-type method for the seven-equation model of compressible two-phase flow. Computers \& Fluids, 54:67-91, 2012.

[4] R. Arienti, P. Hung, E. Morano, and J.E. Shepherd. A level set approach to Eulerian-Lagrangian coupling. J. Comput. Phys., 185:213-251, 2003.

[5] P. Colella, D. T. Graves, B. J. Keen, and D. Modiano. A cartesian grid embedded boundary method for hyperbolic conservation laws. J. Comput. Phys., 211(1):347-366, 2006.

[6] V. Daru and C. Tenaud. High order one-step monotonicity-preserving schemes for unsteady compressible flow calculations. J. Comput. Phys., 193(2):563-594, 2004.

[7] P. De Palma, M. D. De Tullio, G. Pascazio, and M. Napolitano. An immersed-boundary method for compressible viscous flows. Computers $\mathcal{E}$ fluids, 35(7):693-702, 2006. 
[8] J. Donea, S. Giuliani, and J. P Halleux. An arbitrary lagrangian-eulerian finite element method for transient dynamic fluid-structure interactions. Comput. Methods in Appl. Mech. Eng., 33(1):689-723, 1982.

[9] F. Dubois. Lemmes finis pour la dynamique des gaz. http://hal.archives-ouvertes.fr/hal-00733937, 1998.

[10] E. A Fadlun, R. Verzicco, P. Orlandi, and J. Mohd-Yusof. Combined immersed-boundary finitedifference methods for three-dimensional complex flow simulations. J. Comput. Phys., 161(1):35-60, 2000.

[11] J. Falcovitz, G. Alfandary, and G. Hanoch. A two-dimensional conservation laws scheme for compressible flows with moving boundaries. J. Comput. Phys., 138(1):83-102, 1997.

[12] C. Farhat, A. Rallu, and S. Shankaran. A higher-order generalized ghost fluid method for the poor for the three-dimensional two-phase flow computation of underwater implosions. J. Comput. Phys., 227(16):7674-7700, 2008.

[13] N. Favrie, S. L. Gavrilyuk, and R. Saurel. Solid-fluid diffuse interface model in cases of extreme deformations. J. Comput. Phys., 228(16):6037-6077, 2009.

[14] R. P. Fedkiw. Coupling an eulerian fluid calculation to a lagrangian solid calculation with the ghost fluid method. J. Comput. Phys., 175(1):200-224, 2002.

[15] M. A. Fernández, J.-F. Gerbeau, and C. Grandmont. A projection semi-implicit scheme for the coupling of an elastic structure with an incompressible fluid. International Journal for Numerical Methods in Engineering, 69(4):794-821, 2007.

[16] X. Y Hu, B. C. Khoo, N. A. Adams, and F. L. Huang. A conservative interface method for compressible flows. J. Comput. Phys., 219(2):553-578, 2006.

[17] S. R. Idelsohn, J. Marti, A. Limache, and E. Oñate. Unified lagrangian formulation for elastic solids and incompressible fluids: application to fluid-structure interaction problems via the pfem. Comput. Methods in Appl. Mech. Eng., 197(19):1762-1776, 2008.

[18] P. Le Tallec and J. Mouro. Fluid structure interaction with large structural displacements. Comput. Methods in Appl. Mech. Eng., 190(24):3039-3067, 2001.

[19] C. Mariotti and L. Monasse. From general mechanics to discontinuity, unified approach to elasticity. Presses des Ponts, isbn: 978-2-85978-460-7 edition, 2012.

[20] L. Monasse, V. Daru, C. Mariotti, S. Piperno, and C. Tenaud. A conservative coupling algorithm between a compressible flow and a rigid body using an embedded boundary method. J. Comput. Phys., 231(7):2977-2994, 2012.

[21] L. Monasse and C. Mariotti. An energy-preserving discrete element method for elastodynamics. ESAIM, Math. Model. Numer. Anal., 46:1527-1553, 2012.

[22] R. B. Pember, J. B. Bell, P. Colella, W. Y. Crutchfield, and M. L. Welcome. An adaptive cartesian grid method for unsteady compressible flow in irregular regions. J. Comput. Phys., 120(2):278-304, 1995.

[23] C. S. Peskin. Numerical analysis of blood flow in the heart. J. Comput. Phys., 25(3):220-252, 1977.

[24] M. A. Puscas. Development of a numerical coupling method between an inviscid compressible fluid and a deformable structure with possible fragmentation. PhD thesis, University Paris-Est, France, 2014.

[25] M. A. Puscas, V. Daru, A. Ern, C. Mariotti, L. Monasse, and C. Tenaud. Conservative coupling method between an inviscid compressible flow and a deformable structure. Submitted, http://hal.archives-ouvertes.fr/hal-00993324, 2014.

[26] S.K. Sambasivan and H.S. UdayKumar. Ghost Fluid method for strong shock interactions Part 2 : Immersed solid boundaries. AIAA J., 47(12):2923-2937, 2009.

[27] P. Schwartz, M. Barad, P. Colella, and T. Ligocki. A cartesian grid embedded boundary method for the heat equation and poissons equation in three dimensions. J. Comput. Phys., 211(2):531-550, 2006.

[28] A. Soria and F. Casadei. Arbitrary lagrangian-eulerian multicomponent compressible flow with fluid-structure interaction. Int. J. Numer. Methods Fluids, 25(11):1263-1284, 1997.

[29] Y.H. Tseng and J. H. Ferziger. A ghost-cell immersed boundary method for flow in complex geometry. J. Comput. Phys., 192(2):593-623, 2003.

[30] K. Wang, A. Rallu, J. F. Gerbeau, and C. Farhat. Algorithms for interface treatment and load computation in embedded boundary methods for fluid and fluid-structure interaction problems. Int. J. Numer. Methods Fluids, 67(9):1175-1206, 2011. 